TRANSACTIONS OF THE

AMERICAN MATHEMATICAL SOCIETY

Volume 355, Number 4, Pages 1467-1490

S 0002-9947(02)03203-8

Article electronically published on November 22, 2002

\title{
LEBESGUE TYPE DECOMPOSITION OF SUBSPACES OF FOURIER-STIELTJES ALGEBRAS
}

\author{
E. KANIUTH, A. T. LAU, AND G. SCHLICHTING
}

\begin{abstract}
Let $G$ be a locally compact group and let $A(G)$ and $B(G)$ be the Fourier algebra and the Fourier-Stieltjes algebra of $G$, respectively. For any unitary representation $\pi$ of $G$, let $B_{\pi}(G)$ denote the $w^{*}$-closed linear subspace of $B(G)$ generated by all coefficient functions of $\pi$, and $B_{\pi}^{0}(G)$ the closure of $B_{\pi}(G) \cap A_{c}(G)$, where $A_{c}(G)$ consists of all functions in $A(G)$ with compact support. In this paper we present descriptions of $B_{\pi}^{0}(G)$ and its orthogonal complement $B_{\pi}^{s}(G)$ in $B_{\pi}(G)$, generalizing a recent result of T. Miao. We show that for some classes of locally compact groups $G$, there is a dichotomy in the sense that for arbitrary $\pi$, either $B_{\pi}^{0}(G)=\{0\}$ or $B_{\pi}^{0}(G)=A(G)$. We also characterize functions in $\mathcal{B}_{\pi}^{0}(G)=A_{c}(G)+B_{\pi}^{0}(G)$ and study the question of whether $\mathcal{B}_{\pi}^{0}(G)=A(G)$ implies that $\pi$ weakly contains the regular representation.
\end{abstract}

\section{INTRODUCTION}

Let $G$ be a locally compact group, and let $B(G)$ be the Fourier-Stieltjes algebra of $G$ as defined by Eymard [15]. Recall that $B(G)$ is the linear span of all continuous positive definite functions on $G$ and can be identified with the dual of $C^{*}(G)$, the group $C^{*}$-algebra of $G$. The space $B(G)$, with the norm as dual of $C^{*}(G)$, is a commutative Banach $*$-algebra under pointwise multiplication and complex conjugation. The Fourier algebra $A(G)$ is the closed subalgebra of $B(G)$ generated by the functions with compact support. Recall that, when $G$ is abelian, $B(G)$ is isometrically isomorphic (by means of the Fourier transform) to $M(\widehat{G})$, the measure algebra of the dual group $\widehat{G}$ of $G$, and this isomorphism maps $A(G)$ onto $L^{1}(\widehat{G})$.

Generalizing a result of Doss [13] for abelian groups, a Lebesgue type description of $A(G)$ and of its orthogonal complement $B^{s}(G)$ in $B(G)$ for amenable locally compact groups was given by Flory [17, [18] (see also [34, p. 210]). Amenability plays a key role in Flory's proof. Recently Miao [30] established, for an arbitrary locally compact group $G$, an analogous description of $A(G)$ and of $B^{s}(G)$ by viewing $B(G)$ as the dual of $C^{*}(G)$, the group $C^{*}$-algebra, as follows.

Let $u$ be an element of $B(G)$. Then $u$ belongs to $A(G)$ if and only if for every $\epsilon>0$, there is a compact subset $K$ of $G$ such that $|\langle f, u\rangle|<\epsilon$ for all $f \in L^{1}(G)$ with $\|f\|_{C^{*}(G)} \leq 1$ and $\operatorname{supp} f \subseteq G \backslash K$, whereas $u \in B^{s}(G)$ if and only if for each $\epsilon>0$ and each compact subset $K$ of $G$, there exists an $f \in L^{1}(G)$ with

Received by the editors July 9, 2002.

2000 Mathematics Subject Classification. Primary 43A15; Secondary 22D10.

Key words and phrases. Locally compact group, Fourier-Stieltjes algebra, Fourier algebra, unitary representation, coefficient function space, Lebesgue decomposition.

The second author was supported by an NSERC grant. 
$\|f\|_{C^{*}(G)} \leq 1$ and $\operatorname{supp} f \subseteq G \backslash K$ such that $|\langle f, u\rangle| \geq\|u\|-\epsilon$ [30, Corollaries 3.8 and 3.9]. The proof of [30. Theorem 3.7] is fairly complicated and involves an application of Grothendieck's theorem on weakly compact sets in $C(X)$, the space of continuous functions on a compact Hausdorff space $X$, and a careful reduction to second countable groups. Note that Miao's result improves Flory's when $G$ is discrete.

Now, for any representation $\pi$ of $G$, let $B_{\pi}(G)$ denote the $w^{*}$-closed linear subspace of $B(G)$ generated by all coefficient functions of $\pi$. Then $A(G)=$ $\overline{B_{\omega}(G) \cap A_{c}(G)}$, where $\omega$ is the universal representation of $G$ and $A_{c}(G)$ is the space of all functions in $A(G)$ with compact support. It is therefore challenging to study, for arbitrary $\pi$, the subspace $B_{\pi}^{0}(G)=\overline{B_{\pi}(G) \cap A_{c}(G)}$, which is the same as $\overline{B_{\pi}(G) \cap C_{c}(G)}$, of $B_{\pi}(G)$ and its orthogonal complement $B_{\pi}^{s}(G)$ in $B_{\pi}(G)$. In Theorem 2.2 we present descriptions of both $B_{\pi}^{0}(G)$ and $B_{\pi}^{s}(G)$, thus generalizing Miao's result. It is worth pointing out that our proof is extremely elementary. Since the condition identifying $u \in B_{\pi}(G)$ as belonging to $B_{\pi}^{0}(G)$ is also satisfied by functions in $A_{c}(G)$, an equally interesting subspace of $B(G)$ is $\mathcal{B}_{\pi}^{0}(G)=A_{c}(G)+B_{\pi}^{0}(G)$. Theorem 2.4 gives an analogous description of $\mathcal{B}_{\pi}^{0}(G)$.

The major portion of the paper, however, is devoted to the study of how $B_{\pi}^{0}(G)$ and $\mathcal{B}_{\pi}^{0}(G)$ depend on $\pi$ and on $G$. To begin with, we collect in Section 3 a number of basic properties of $B_{\pi}^{0}(G)$ and $\mathcal{B}_{\pi}^{0}(G)$. It is clear that $B_{\pi}^{0}(G)=A(G)$ precisely when $\pi$ weakly contains the regular representation $\rho$ of $G$. In Section 4 we focus on the question of whether the weaker condition that $\mathcal{B}_{\pi}^{0}(G)=A(G)$ already implies that $\pi$ weakly contains $\rho$. This appears to be a difficult problem. We obtain several results (Theorem 4.2, Theorem 4.9 and Corollaries 4.10 and 4.11) which strongly support the conjecture that this question has an affirmative answer in general.

It turns out that for some classes of locally compact groups $G$ there is a dichotomy in the sense that, for an arbitrary representation $\pi$ of $G$, either $B_{\pi}^{0}(G)=\{0\}$ or $B_{\pi}^{0}(G)=A(G)$. Most notably, this holds for groups with small invariant neighbourhoods of the identity (SIN-groups) (Theorem 5.6) and also for various other groups (see Proposition 5.3 and Examples 5.4). Finally, in Section 6 we conclude with some more specific results on $B_{\pi}^{0}(G)$ for irreducible representations $\pi$ of nilpotent groups.

Various other geometric and topological properties of the Fourier-Stieltjes algebra and of the spaces $B_{\pi}(G)$ have been studied by several authors (compare [2], [3], 4], [5], 7], [9], 19], 27], 28], 29] and [37, and the references therein).

\section{Preliminaries}

Let $G$ be a locally compact group with a fixed left Haar measure. As usual, we denote by $C^{*}(G)$ the completion of the convolution algebra $L^{1}(G)$ with respect to the norm $\|f\|_{C^{*}(G)}=\sup \{\|\pi(f)\|\}$, where the supremum is taken over all *representations $\pi$ of $L^{1}(G)$ as an algebra of bounded linear operators in a Hilbert space. Let $P(G)$ be the set of all continuous positive definite functions on $G$, and let $B(G)$ denote the linear span of $P(G)$. Then $B(G)$ can be identified with the dual space $C^{*}(G)^{*}$ of $C^{*}(G)$ by the pairing $\langle f, u\rangle=\int_{G} f(x) u(x) d x$ for $f \in L^{1}(G)$ and $u \in$ $B(G)$. With pointwise multiplication and the dual norm, $B(G)$ is a commutative Banach algebra, called the Fourier-Stieltjes algebra. The Fourier algebra $A(G)$ is the closure in $B(G)$ of $A_{c}(G)=B(G) \cap C_{c}(G)$, the compactly supported functions in $B(G)$. For any compact subset $K$ of $G$, we denote by $A_{K}(G)$ the set of functions 
$u \in A(G)$ with supp $u \subseteq K$. Then $A(G)$ is a closed ideal in $B(G)$. For details regarding $B(G)$ and $A(G)$, see the fundamental paper [15].

As is customary, we shall use the same letter, for example $\pi$, for a unitary representation of $G$ and the corresponding *-representations of $L^{1}(G)$ and $C^{*}(G)$. For any representation $\pi$ of $G$, let $A_{\pi}(G)$ denote the closed linear subspace of $B(G)$ generated by all coefficient functions of $\pi$, and $B_{\pi}(G)$ the $w^{*}$-closure of $A_{\pi}(G)$ in $B(G)=C^{*}(G)^{*}$. Then $B_{\pi}(G)$ can be identified with the dual of $\pi\left(C^{*}(G)\right)$. Note that, when $\rho=\rho_{G}$ denotes the regular representation of $G$ on $L^{2}(G)$, then $A_{\rho}(G)=A(G)$, and $B_{\rho}(G)$ equals $B(G)$ if and only if $G$ is amenable. Concerning the theory of amenable groups, we refer to the monographs [33] and [34. Also, $A(G)$ is the unique predual of the von Neumann algebra generated by all operators $\rho(f), f \in L^{1}(G)$, the pairing being given by $\langle u, \rho(f)\rangle=\int_{G} u(x) f(x) d x$.

If $S$ and $T$ are sets of unitary representations of $G$, then $S$ is weakly contained in $T(S \prec T)$, if $\bigcap\{\operatorname{ker} \sigma: \sigma \in S\} \supseteq \bigcap\{\operatorname{ker} \tau: \tau \in T\}$, where ker $\pi$ denotes the $C^{*}$-kernel of a representation $\pi . S$ and $T$ are said to be weakly equivalent $(S \sim T)$ if $S \prec T$ and $T \prec S$. For any two representations $\sigma$ and $\pi, \sigma \prec \pi$ if and only if $B_{\sigma}(G) \subseteq B_{\pi}(G)$ and if and only if $\|\sigma(f)\| \leq\|\pi(f)\|$ for all $f \in L^{1}(G)$. We shall frequently use throughout the paper the fact that if $u \in B_{\pi}(G)$, then there exists a representation $\sigma$ of $G$ such that $\sigma \prec \pi$ and $u$ is a coordinate function of $\sigma$. Indeed, this follows from [1 Théorème 3.17 and Théorème 2.2]. The dual space $\widehat{G}$ of $G$ consists of all equivalence classes of irreducible unitary representations of $G$ and is topologized so that $\tau \in \widehat{G}$ belongs to the closure of a subset $S$ of $\widehat{G}$ if and only if $\tau \prec S$. For an arbitrary representation $\pi$ of $G$, the support of $\pi, \operatorname{supp} \pi$, is the closed subset of all $\tau \in \widehat{G}$ such that $\tau \prec \pi$. Thus $\pi \sim \widehat{G}$ precisely when $\operatorname{supp} \pi=\widehat{G}$. As general references to duals of locally compact groups and weak containment properties, we mention [11] and [16].

Let $H$ be a closed subgroup of $G$ and $\tau$ a unitary representation of $H$. The representation of $G$ induced by $\tau$ is denoted $\operatorname{ind}_{H}^{G} \tau$. In Sections 5 and 6 we shall use the fact that $\pi \prec \operatorname{ind}_{H}^{G}(\pi \mid H)$ for any representation $\pi$ of $G$ whenever $G$ is amenable [20, Theorem 5.1].

A locally compact group is called an SIN-group (a group with small invariant neighbourhoods) if there exists a neighbourhood basis of the identity consisting of sets $V$ such that $x^{-1} V x=V$ for all $x \in G$. SIN-groups provide a large class of locally compact groups comprising all abelian groups, compact groups and discrete groups. For the structure theory of SIN-groups, see [21].

Let $A$ be a $C^{*}$-algebra. For elements $S \in A$ and $\phi \in A^{*}$ we define the Sakai products $S \cdot \phi$ and $\phi \cdot S$ in $A^{*}$ by $\langle S \cdot \phi, T\rangle=\langle\phi, T S\rangle$ and $\langle\phi \cdot S, T\rangle=\langle\phi, S T\rangle$ for every $T \in A$. A subset $V$ of $A^{*}$ is called invariant if $S \cdot V \subseteq V$ and $V \cdot S \subseteq V$ for every $S \in A$.

Note that if $G$ is a locally compact group and $X$ is a closed subspace of $B(G)$, then $X$ is invariant as a subspace of $B(G)=C^{*}(G)^{*}$ if and only if $X$ is two-sided translation invariant under the action of $G$ (see [36, p. 123, Theorem 2.7]). In this case, $X=A_{\sigma}(G)$ for some unitary representation $\sigma$ of $G$ [1, Théorème 3.17].

If $\mathcal{M}$ is a von Neumann algebra, the unique predual $\mathcal{M}_{*}$ of $\mathcal{M}$ is invariant, regarded as a subspace of $\mathcal{M}^{*}$.

Furthermore, there is a one-to-one correspondence between closed invariant subspaces $V$ of $M_{*}$ and $w^{*}$-closed ideals $I$ in $M$, given by $V^{\perp}=I$ and $I^{\perp}=V$, where $V^{\perp}$ and $I^{\perp}$ mean the annihilator of $V$ and $I$ in $M$ and $M_{*}$, respectively. 
Every closed invariant subspace $V$ of $M_{*}$ is of the form $V=E \cdot M_{*}=M_{*} \cdot E$ for a unique central projection $E$ in $M$. Hence there is a direct sum decomposition $M_{*}=V \oplus(I-E) \cdot M_{*}$ (see [36] p. 123]). Also, using polar decomposition of $u \in M_{*}$ (see [36, p. 140]), it follows readily that $\|u\|=\|E \cdot u\|+\|(I-E) \cdot u\|$.

\section{Generalizations of MiaO's Decomposition theorem}

Recently Miao [30] has proved the following Lebesgue type decomposition theorem for the Fourier-Stieltjes algebra.

Theorem 2.1 (30]). Let $G$ be a locally compact group and let $u \in B(G)$. Let $E$ denote the central projection in the von Neumann algebra $B(G)^{*}$ such that $A(G)=$ $E \cdot B(G)$. Then:

(i) $u \in A(G)$ if and only if for every $\epsilon>0$, there exists a compact subset $K$ of $G$ such that $|\langle u, f\rangle|<\epsilon$ for all $f \in L^{1}(G \backslash K)$ with $\|f\|_{C^{*}(G)} \leq 1$.

(ii) $u \in(I-E) \cdot B(G)$ if and only if for every $\epsilon>0$ and each compact subset $K$ of $G$, there exists $f \in L^{1}(G \backslash K)$ such that $\|f\|_{C^{*}(G)} \leq 1$ and $|\langle u, f\rangle|>\|u\|-\epsilon$.

Let $\omega$ denote the universal representation of $G$. Then $B_{\omega}(G)=B(G)$ and $\|f\|_{C^{*}(G)}=\|f\|_{\omega}$ for all $f \in L^{1}(G)$. Also, $A(G)=\overline{A_{c}(G)}=\overline{B_{\omega}(G) \cap A_{c}(G)}$. This leads us to introduce, for an arbitrary representation $\pi$ of $G$, two subspaces of $B_{\pi}(G)$ as follows. First, let

$$
B_{\pi}^{0}(G)=\overline{B_{\pi}(G) \cap A_{c}(G)} .
$$

Since $B_{\pi}^{0}(G)$ is a closed translation-invariant subspace of $B_{\pi}(G)$, there exists a central projection $E$ in $B_{\pi}(G)^{*}$ such that $B_{\pi}^{0}(G)=E \cdot B_{\pi}(G)$. Let

$$
B_{\pi}^{s}(G)=(I-E) \cdot B_{\pi}(G) .
$$

Thus $B_{\pi}(G)=B_{\pi}^{0}(G) \oplus B_{\pi}^{s}(G)$. Then $B_{\pi}^{s}(G)$ is the "orthogonal" complement of $B_{\pi}^{0}(G)$ in $B_{\pi}(G)$ in the sense that if $u=v+w$, where $v \in B_{\pi}^{0}(G)$ and $w \in B_{\pi}^{s}(G)$, then $\|u\|=\|v\|+\|w\|$.

The first purpose of this section is to present descriptions of both $B_{\pi}^{0}(G)$ and $B_{\pi}^{s}(G)$ analogous to those of Theorem 2.1. First, however, we need some notation.

Let $\mathcal{K}$ denote the set of all compact subsets of $G$. To any representation $\pi$ of $G$ and any $K \in \mathcal{K}$, we associate a seminorm $\|\cdot\|_{\pi, K}: B(G) \rightarrow[0, \infty]$ by

$$
\|u\|_{\pi, K}=\sup \left\{|\langle u, f\rangle|: f \in L^{1}(G \backslash K),\|\pi(f)\| \leq 1\right\} .
$$

Directing $\mathcal{K}$ by upward inclusion, we have $\|u\|_{\pi, K_{1}} \leq\|u\|_{\pi, K_{2}}$ whenever $K_{1} \geq K_{2}$. Thus the limit

$$
\|u\|_{\pi}=\lim _{K}\|u\|_{\pi, K}=\inf \left\{\|u\|_{\pi, K}: K \in \mathcal{K}\right\}
$$

exists.

Theorem 2.2. Let $G$ be a locally compact group, $\pi$ a unitary representation of $G$ and $u \in B_{\pi}(G)$. Then:

(i) $u \in B_{\pi}^{0}(G)$ if and only if for every $\epsilon>0$ there exists a compact subset $K$ of $G$ such that $|\langle u, f\rangle|<\epsilon$ for all $f \in L^{1}(G \backslash K)$ with $\|\pi(f)\| \leq 1$.

(ii) $u \in B_{\pi}^{s}(G)$ if and only if for every $\epsilon>0$ and each compact subset $K$ of $G$ there exists $f \in L^{1}(G \backslash K)$ such that $|\langle u, f\rangle|>\|u\|-\epsilon$ and $\|\pi(f)\| \leq 1$. 
Proof. Notice that $\|u\|_{\pi}=0$ if and only if $u$ satisfies the condition in (i), whereas the condition in (ii) is equivalent to $\|u\|_{\pi}=\|u\|$.

Regarding $L^{1}(G \backslash K)$, with respect to the seminorm $f \rightarrow\|\pi(f)\|$, as a (nonclosed) subspace of $C^{*}(G)$, by the Hahn-Banach theorem it is clear that

$$
\|u\|_{\pi, K}=\left\|u \mid L^{1}(G \backslash K)\right\|=\operatorname{dist}\left(u, L^{1}(G \backslash K)^{\perp}\right),
$$

where the annihilator is taken in $B_{\pi}(G)$. Since $L^{1}(G \backslash K)^{\perp}=A_{K}(G) \cap B_{\pi}(G)$, we get

$$
\begin{aligned}
\inf _{K} \operatorname{dist}\left(u, A_{K}(G)\right. & \left.\cap B_{\pi}(G)\right)=\operatorname{dist}\left(u, \bigcup_{K \in \mathcal{K}}\left(A_{K}(G) \cap B_{\pi}(G)\right)\right) \\
& =\operatorname{dist}\left(u, A_{c}(G) \cap B_{\pi}(G)\right) .
\end{aligned}
$$

Hence, by (1),

$$
\|u\|_{\pi}=\operatorname{dist}\left(u, A_{c}(G) \cap B_{\pi}(G)\right) .
$$

In particular, $\|u\|_{\pi}=0$ if and only if $u \in B_{\pi}^{0}(G)$. This proves (i).

For (ii), since $B_{\pi}^{s}(G)$ is the orthogonal complement of $B_{\pi}^{0}(G)$ in $B_{\pi}(G)$, the above distance formula yields that $u \in(I-E) \cdot B_{\pi}(G)$ if and only if

$$
\|u\|=\|(I-E) u\|=\operatorname{dist}\left((I-E) u, B_{\pi}^{0}(G)\right)=\operatorname{dist}\left(u, B_{\pi}^{0}(G)\right)=\|u\|_{\pi} .
$$

Hence

$$
(I-E) \cdot B_{\pi}(G)=\left\{u \in B_{\pi}(G):\|u\|=\|u\|_{\pi}\right\},
$$

as was to be shown.

In particular, Theorem 2.2 provides, taking $\pi=\omega$, an extremely simple proof of Miao's theorem. However, our method does not seem to apply to give a proof of Flory's theorem.

It is obvious that if $u$ is a compactly supported function in $B(G)$ (that is, $u \in$ $A_{c}(G)$ ), then $u$ satisfies the condition in (i) of Theorem 2.2 no matter what $\pi$ is. We are going to show next that this condition holds precisely for the functions in $A_{c}(G)+B_{\pi}^{0}(G)$.

Lemma 2.3. Let $G$ be a locally compact group, and let $u \in B(G)$ and $K \in \mathcal{K}$. Then the follwing are equivalent.

(i) $\|u\|_{\pi, K}<\infty$.

(ii) The restriction of $u$ to a linear functional on $L^{1}(G \backslash K)$ is continuous with respect to the seminorm $f \rightarrow\|\pi(f)\|$.

(iii) There is a $u_{K} \in B_{\pi}(G)$ such that $u\left|L^{1}(G \backslash K)=u_{K}\right| L^{1}(G \backslash K)$.

(iv) There exists $u_{K} \in B_{\pi}(G)$ such that $u-u_{K} \in A_{K}(G)$.

(v) $\left(A_{K}(G)+u\right) \cap B_{\pi}(G) \neq \emptyset$.

(vi) $u \in B_{\pi}(G)+A_{K}(G)$.

Proof. The equivalences (i) $\Leftrightarrow$ (ii) and (iii) $\Leftrightarrow$ (iv) $\Leftrightarrow$ (v) $\Leftrightarrow$ (vi) and the implication (iii) $\Rightarrow$ (ii) are trivial. Also, (ii) $\Rightarrow$ (iii) follows from the Hahn-Banach extension theorem.

Theorem 2.4. Let $G$ be a locally compact group and $\pi$ any representation of $G$. Then for $u \in B(G)$ the following conditions are equivalent.

(i) $u \in A_{c}(G)+B_{\pi}^{0}(G)$.

(ii) For every $\epsilon>0$ there exists a compact subset $K$ of $G$ such that $|\langle u, f\rangle|<\epsilon$ for all $f \in L^{1}(G \backslash K)$ with $\|\pi(f)\| \leq 1$. 
Proof. Using Theorem 2.2, we only have to show that if $u$ satisfies condition (ii), then $u \in A_{c}(G)+B_{\pi}^{0}(G)$. Let $K \in \mathcal{K}$ be such that $\|u\|_{\pi, K}<\infty$ and let $u_{K} \in B_{\pi}(G)$ be such that $u\left|L^{1}(G \backslash K)=u_{K}\right| L^{1}(G \backslash K)$ (Lemma 2.3). Then

$$
\begin{aligned}
\|u\|_{\pi, K} & =\left\|u_{K} \mid L^{1}(G \backslash K)\right\|=\operatorname{dist}\left(u_{K}, L^{1}(G \backslash K)^{\perp} \cap B_{\pi}(G)\right) \\
& =\operatorname{dist}\left(0,\left(A_{K}(G) \cap B_{\pi}(G)\right)-u_{K}\right)=\operatorname{dist}\left(0,\left(A_{K}(G)-u_{K}\right) \cap B_{\pi}(G)\right) \\
& =\operatorname{dist}\left(0,\left(A_{K}(G)-u\right) \cap B_{\pi}(G)\right) .
\end{aligned}
$$

In particular, (ii) holds for $u$ if and only if $0 \in \overline{\left(A_{c}(G)-u\right) \cap B_{\pi}(G)}$. Since, by Lemma 2.3 , (i) $\Leftrightarrow$ (vi), $u \in A_{c}(G)+B_{\pi}(G)$, it follows that (ii) holds for $u$ precisely when

$$
\begin{gathered}
u \in A_{c}(G)+\left\{v \in B_{\pi}(G): 0 \in \overline{\left(A_{c}(G)-v\right) \cap B_{\pi}(G)}\right\} \\
=A_{c}(G)+\left\{v \in B_{\pi}(G): 0 \in \overline{A_{c}(G) \cap B_{\pi}(G)}-v\right\} \\
=A_{c}(G)+B_{\pi}^{0}(G) .
\end{gathered}
$$

From now on we set, for an arbitrary representation $\pi$ of $G$,

$$
\mathcal{B}_{\pi}^{0}(G)=A_{c}(G)+B_{\pi}^{0}(G) .
$$

Let $\pi$ and $\sigma$ be representations of $G$ that are weakly equivalent. It follows from Theorem 2.2(i) that $B_{\pi}^{0}(G)=B_{\sigma}^{0}(G)$, and hence also $\mathcal{B}_{\pi}^{0}(G)=\mathcal{B}_{\sigma}^{0}(G)$.

We conclude this section with a number of corollaries. First, we restate Theorems 2.2 and 2.4 with $\pi$ the regular representation $\rho$.

Corollary 2.5. (i) $\mathcal{B}_{\rho}^{0}(G)=A(G)=B_{\rho}^{0}(G)$.

(ii) $B_{\rho}^{s}(G)$ is the orthogonal complement of $A(G)$ in $B_{\rho}(G)$.

(iii) $B_{\rho}(G)=A(G) \oplus B_{\rho}^{s}(G)$ (orthogonal direct sum).

Corollary 2.6. For a representation $\pi$ of $G$ the following conditions are equivalent.

(i) $\mathcal{B}_{\pi}^{0}(G) \subseteq B_{\pi}(G)$.

(ii) $\left\{u \in B(G):\|u\|_{\pi}<\infty\right\} \subseteq B_{\pi}(G)$.

(iii) $\pi$ weakly contains the regular representation.

Proof. This is an immediate consequence of Lemma 2.3, Theorem 2.4 and the two facts that $B_{\rho}(G)$ is the $w^{*}$-closure of $A(G)$ and that $\pi$ weakly contains $\rho$ if and only if $B_{\pi}(G) \supseteq B_{\rho}(G)$.

Let $\mathcal{B}_{\pi}^{\infty}(G)=\left\{u \in B(G):\|u\|_{\pi}<\infty\right\}$. Clearly, $B_{\pi}(G) \subseteq \mathcal{B}_{\pi}^{\infty}(G)$ and, according to the preceding corollary, the converse inclusion holds if and only if $\pi$ weakly contains the regular representation. In general, we have the following.

Corollary 2.7. Let $\pi$ be a representation of $G$. Then:

(i) $\mathcal{B}_{\pi}^{\infty}(G)=A_{c}(G)+B_{\pi}(G)=\mathcal{B}_{\pi}^{0}(G) \oplus B_{\pi}^{s}(G)$ (algebraic direct sum).

(ii) $\mathcal{B}_{\pi}^{0}(G)$ is closed in $\mathcal{B}_{\pi}^{\infty}(G)$, and the quotient space $\mathcal{B}_{\pi}^{\infty}(G) / \mathcal{B}_{\pi}^{0}(G)$ is isometrically isomorphic to $B_{\pi}^{s}(G)$.

Proof. (i) Since $\|u\|_{\pi}<\infty$ if and only if $\|u\|_{\pi, K}<\infty$ for some $K \in \mathcal{K}$, the first equality follows immediately from Lemma 2.3. Then, by the definition of $\mathcal{B}_{\pi}^{0}(G)$,

$$
\mathcal{B}_{\pi}^{0}(G)+B_{\pi}^{s}(G)=A_{c}(G)+B_{\pi}^{0}(G)+B_{\pi}^{s}(G)=A_{c}(G)+B_{\pi}(G)=\mathcal{B}_{\pi}^{\infty}(G),
$$


and the sum on the left-hand side is direct, since $\|u\|_{\pi}=\|u\|$ for $u \in B_{\pi}(G)$ and $\|u\|_{\pi}=0$ for $u \in \mathcal{B}_{\pi}^{0}(G)$.

(ii) The seminorm $\|\cdot\|_{\pi}$ on $\mathcal{B}_{\pi}^{\infty}(G)$ defines a norm $|\cdot|$ on the quotient space $\mathcal{B}_{\pi}^{\infty}(G) / \mathcal{B}_{\pi}^{0}(G)$ by $\left|u+\mathcal{B}_{\pi}^{0}(G)\right|=\|u\|_{\pi}, u \in \mathcal{B}_{\pi}^{\infty}(G)$. So $u \rightarrow u+\mathcal{B}_{\pi}^{0}(G)$ is an isometric isomorphism between $B_{\pi}^{s}(G)$ and $\mathcal{B}_{\pi}^{\infty}(G) / \mathcal{B}_{\pi}^{0}(G)$.

\section{General properties of $B_{\pi}^{0}(G)$ And $\mathcal{B}_{\pi}^{0}(G)$}

In this section we collect a number of basic properties of $B_{\pi}^{0}(G)$ and of $\mathcal{B}_{\pi}^{0}(G)$, which will be used in the subsequent sections.

As mentioned above, the subspaces $B_{\pi}^{0}(G)$ and $\mathcal{B}_{\pi}^{0}(G)$ depend only on ker $\pi$. Slightly more generally, we have the following lemma, which follows immediately from Theorems 2.2 and 2.4 and the fact that $\|\pi(f)\| \geq\|\sigma(f)\|$ for all $f \in L^{1}(G)$ whenever the representation $\sigma$ is weakly contained in $\pi$.

Lemma 3.1. Let $\pi$ and $\sigma$ be representations of $G$ such that $\sigma$ is weakly contained in $\pi$. Then $B_{\pi}^{0}(G) \supseteq B_{\sigma}^{0}(G)$ and $\mathcal{B}_{\pi}^{0}(G) \supseteq \mathcal{B}_{\sigma}^{0}(G)$.

Lemma 3.2. Let $G$ be a non-compact locally compact group and $\pi$ any representation of $G$. Then $\mathcal{B}_{\pi}^{0}(G)=A_{c}(G)$ if and only if $B_{\pi}(G) \cap A_{c}(G)=\{0\}$. In particular, $B_{\pi}^{0}(G)=\{0\}$ if and only if $\mathcal{B}_{\pi}^{0}(G)=A_{c}(G)$.

Proof. We first note that $A_{c}(G)$ does not contain any invariant closed subspace $V \neq\{0\}$ of $B(G)$. Otherwise, let $v \in V, v \neq 0$. Then $K=\operatorname{supp} v$ is compact, and since $G$ is non-compact, there exist $a_{j} \in G, j \in \mathbb{N}$, such that $a_{j} K \cap a_{i} K=\emptyset$ for $j \neq i$. Let

$$
u=\sum_{j=1}^{\infty} 2^{-j} L_{a_{j}} v \in B(G) .
$$

Then $u \in V$, but $\operatorname{supp} u$ is not compact, a contradiction. Now, by definition, $\mathcal{B}_{\pi}^{0}(G)=A_{c}(G)$ if and only if $B_{\pi}^{0}(G) \subseteq A_{c}(G)$, and hence $\mathcal{B}_{\pi}^{0}(G)=A_{c}(G)$ if and only if $A_{c}(G) \cap B_{\pi}(G)=\{0\}$.

The last statement follows from the definition.

Lemma 3.3. Let $\pi$ be any representation of $G$, and suppose that $B_{\pi}(G) \cap A(G) \neq$ $\{0\}$. Then

$$
P K_{\pi}=\left\{x \in G: \pi(x) \in \mathbb{T} \cdot I_{\mathcal{H}_{\pi}}\right\},
$$

the projective kernel of $\pi$, is compact.

Proof. Let $\phi$ be a positive definite function on $G$ such that $\phi(e)=1$, and let $G_{1}=\{x \in G:|\phi(x)|=1\}$. Then $\phi(x y)=\phi(x) \phi(y)$ for all $x \in G_{1}$ and $y \in G$ [23, Corollary 32.7]. Thus, if $\phi$ is associated to $\pi$, then $\phi(x y)=\chi(x) \phi(y)$ for all $y \in G$ and $x \in P K_{\pi}$, where $\chi(x) \in \mathbb{T}$. It follows that if $u$ is a linear combination of normalized positive definite functions associated to $\pi$, then $u(x y)=\chi(x) u(y)$ for all $y \in G$ and $x \in P K_{\pi}$. This formula is preserved under taking $w^{*}$-limits; hence it holds for all $u \in B_{\pi}(G), y \in G$ and $x \in P K_{\pi}$.

Now, let $u \in B_{\pi}(G) \cap A(G), u \neq 0$, and fix $y \in G$ such that $u(y) \neq 0$. Then, since $u \in C_{0}(G)$, the function $x \rightarrow u(x y)=\chi(x) u(y)$ vanishes at infinity on $P K_{\pi}$. Consequently, $P K_{\pi}$ has to be compact.

Corollary 3.4. Let $G$ be a locally compact group with non-compact centre. Then $B_{\pi}^{0}(G)=\{0\}$ for every irreducible representation $\pi$ of $G$. 
Lemma 3.5. If $\pi$ is a finite-dimensional representation of a non-compact locally compact group $G$, then $B_{\pi}^{0}(G)=\{0\}$, and hence $\mathcal{B}_{\pi}^{0}(G)=A_{c}(G)$.

Proof. Suppose that $B_{\pi}^{0}(G) \neq\{0\}$. Then $B_{\pi}(G) \cap A(G)$ is a finite-dimensional non-trivial invariant subspace of $C_{0}(G)$, that is, $C_{0}(G)$ contains a nonzero almost periodic function. This implies that $G$ is compact [8, Corollary 3.9], a contradiction. The second statement follows from the definition of $\mathcal{B}_{\pi}^{0}(G)$.

Lemma 3.6. Let $\pi$ be an irreducible representation of $G$, and suppose that the singleton $\{\pi\}$ is closed in $\widehat{G}$ and that $\pi$ is not square integrable. Then $B_{\pi}^{0}(G)=\{0\}$, and hence $\mathcal{B}_{\pi}^{0}(G)=A_{c}(G)$.

Proof. That $\{\pi\}$ is closed in $\widehat{G}$ implies that $A_{\pi}(G)=B_{\pi}(G)$. Indeed, if $u \in B_{\pi}(G)$, then $u$ is a coordinate function of some representation $\sigma$ that is weakly contained in $\pi$. Now, since $\{\pi\}$ is closed in $\widehat{G}, \sigma$ is a multiple of $\pi$, and this implies that $u \in A_{\pi}(G)$. The fact that $\pi$ is not square integrable yields that $A(G) \cap A_{\pi}(G)=\{0\}$; otherwise $\pi$ would be equivalent to a subrepresentation of $\rho$. Thus $B_{\pi}(G) \cap A(G)=$ $\{0\}$.

Actually, it is easy to see that for an irreducible representation $\pi$ of $G,\{\pi\}$ is closed in $\widehat{G}$ if and only if $A_{\pi}(G)=B_{\pi}(G)$.

Lemma 3.7. Let $H$ be a closed subgroup of $G$ and $\pi$ a representation of $G$. Then $\mathcal{B}_{\pi}^{0}(G) \mid H \subseteq \mathcal{B}_{\pi \mid H}^{0}(H)$ and $B_{\pi}^{0}(G) \mid H \subseteq B_{\pi \mid H}^{0}(H)$. In particular, if $\mathcal{B}_{\pi}^{0}(G)=A(G)$, then $\mathcal{B}_{\pi \mid H}^{0}(H)=A(H)$, and if $B_{\pi}^{0}(G)=A(G)$, then $B_{\pi \mid H}^{0}(H)=A(H)$.

Proof. Since $A_{c}(G) \mid H \subseteq A_{c}(H)$ and $\mathcal{B}_{\pi}^{0}(G)=A_{c}(G)+B_{\pi}^{0}(G)$, to establish the two inclusions it is enough to show that $B_{\pi}^{0}(G) \mid H \subseteq B_{\pi \mid H}^{0}(H)$. To this end, select a representation $\tau$ of $G$ such that $\tau \sim \pi$ and $B_{\pi}(G)=A_{\tau}(G)$. Now, $A_{\tau}(G) \mid H \subseteq$ $A_{\tau \mid H}(H)$ and $\tau \mid H$ is weakly equivalent to $\pi \mid H$. Since the restriction map from $B(G)$ to $B(H)$ is norm continuous, we therefore obtain

$$
\begin{aligned}
& B_{\pi}^{0}(G)\left|H=\left(\overline{A_{\tau}(G) \cap A_{c}(G)}\right)\right| H \subseteq \overline{A_{\tau \mid H}(H) \cap A_{c}(H)} \\
& \subseteq \overline{B_{\tau \mid H}(H) \cap A_{c}(H)}=\overline{B_{\pi \mid H}(H) \cap A_{c}(H)}=B_{\pi \mid H}^{0}(H) .
\end{aligned}
$$

Since $A(G) \mid H=A(H)$ (see [22] and [1, Proposition 3.23]), the last statement of the lemma follows also.

In general, as the following lemma shows, there are examples of groups $G$ and representations $\pi$ such that

$$
A_{c}(G) \subsetneq \mathcal{B}_{\pi}^{0}(G) \subsetneq A(G) .
$$

Lemma 3.8. Let $G$ be a non-compact locally compact group and $K$ a non-trivial compact normal subgroup of $G$, and let $\pi=\rho \circ q$, where $\rho$ is the regular representation of $G / K$ and $q$ is the quotient homomorphism $G \rightarrow G / K$. Then

$$
A_{c}(G) \subsetneq \mathcal{B}_{\pi}^{0}(G) \subsetneq A(G),
$$

and therefore also $\{0\} \neq B_{\pi}^{0}(G) \neq A(G)$.

Proof. Notice first that $A(G / K) \circ q \subseteq B_{\rho}(G / K) \circ q=B_{\pi}(G)$. Since $G$ is noncompact, $A(G / K) \circ q \nsubseteq A_{c}(G)$, whence $A_{c}(G)$ is properly contained in $A_{c}(G)+$ $\overline{B_{\pi}(G) \cap A(G)}=\mathcal{B}_{\pi}^{0}(G)$. Suppose that $\mathcal{B}_{\pi}^{0}(G)=A(G)$, so that $A(G)=A_{c}(G)+$ 
$A(G / K) \circ q$. By adapting the argument in the proof of Lemma 3.2, we show that this is impossible. Since $K$ is non-trivial, we can choose $w_{0} \in A_{c}(G)$ such that $W=\operatorname{supp} w_{0}$ satisfies $e \in W$ and $W \cap K \neq K$. Since $G$ is non-compact, we find $a_{j} \in G, j \in \mathbb{N}$, such that $a_{j} W K \cap a_{i} W K=\emptyset$ whenever $j \neq i$. Let

$$
w=\sum_{j=1}^{\infty} 2^{-j} L_{a_{j}} w_{0} \in A(G) .
$$

By hypothesis, $w=u+v$, where $u \in A_{c}(G)$ and $v \in A(G / K) \circ q$. Since $u$ has compact support, it follows that, for some $N \in \mathbb{N}, w(x)=v(x)$ for all $x \in$ $\bigcup_{j=N}^{\infty} a_{j} W K$. Now $v$ is constant on cosets of $K$, whereas for each $j$,

$$
\operatorname{supp} w \cap a_{j} K=\operatorname{supp}\left(L_{a_{j}} w_{0}\right) \cap a_{j} K=a_{j}\left(\operatorname{supp} w_{0} \cap K\right) \subsetneq a_{j} K .
$$

This contradiction shows that $\mathcal{B}_{\pi}^{0}(G) \neq A(G)$.

Let $N$ be a compact normal subgroup of $G$, and let $\pi$ be a representation of $G$ on the Hilbert space $\mathcal{H}_{\pi}$. As in [30, Proposition 3.4], we can associate to $\pi$ a representation $\pi_{N}$ of the quotient group $G / N$ as follows, provided that $\pi \mid N$ contains the trivial representation of $N$. Let $d t$ denote normalized Haar measure on $N$, and let Haar measures on $G$ and $G / N$ be normalized so that Weil's formula holds. Define a selfadjoint projection $P$ in $\mathcal{H}_{\pi}$ by

$$
\langle P \xi, \eta\rangle=\int_{N}\langle\pi(x t) \xi, \eta\rangle d t, \quad \xi, \eta \in \mathcal{H}_{\pi} .
$$

Obviously, $P\left(\mathcal{H}_{\pi}\right)$ consists precisely of all fixed vectors in $\mathcal{H}_{\pi}$ under the action of all operators $\pi(t), t \in N$, and therefore $P\left(\mathcal{H}_{\pi}\right) \neq\{0\}$ if and only if $\pi \mid N$ contains the trivial representation of $N$. In this case $\pi_{N}$, defined by

$$
\left\langle\pi_{N}(x N) \xi, \eta\right\rangle=\int_{N}\langle\pi(x t) \xi, \eta\rangle d t, \quad \xi, \eta \in P\left(\mathcal{H}_{\pi}\right),
$$

is a unitary representation of $G / N$ on $P\left(\mathcal{H}_{\pi}\right)$. Moreover, there is a canonical linear map $\phi: u \rightarrow u_{N}$ from $B(G)$ onto $B(G / N)$ given by

$$
u_{N}(x N)=\int_{N} u(x t) d t, x \in G
$$

Lemma 3.9. $\phi$ maps $B_{\pi}^{0}(G)$ onto $B_{\pi_{N}}^{0}(G / N)$ and $\mathcal{B}_{\pi}^{0}(G)$ onto $\mathcal{B}_{\pi_{N}}^{0}(G / N)$.

Proof. Let $q: G \rightarrow G / N$ denote the quotient homomorphism, and let $u \in B_{\pi}^{0}(G)$ and $\epsilon>0$ be given. By Theorem 2.2(i), there exists a compact subset $K$ of $G$ such that $K=q^{-1}(q(K))$ and $|\langle u, f\rangle|<\epsilon$ for all $f \in L^{1}(G \backslash K)$ with $\|\pi(f)\| \leq 1$. Let $g \in L^{1}(G / N \backslash q(K))$ with $\left\|\pi_{N}(g)\right\| \leq 1$. Then $f=g \circ q \in L^{1}(G \backslash K)$ and $\langle u, f\rangle=\left\langle u_{N}, g\right\rangle$. In addition, using the properties of $P$ (see the proof of 30 . Proposition 3.4]), it is easily verified that

$$
\langle\pi(f) \xi, \eta\rangle=\left\langle\pi_{N}(g) P \xi, P \eta\right\rangle
$$

for all $\xi, \eta \in \mathcal{H}_{\pi}$. Thus, if $\|\xi\|,\|\eta\| \leq 1$, then $|\langle\pi(f) \xi, \eta\rangle| \leq\left\|\pi_{N}(g)\right\| \leq 1$. It follows that $\|\pi(f)\| \leq 1$, and hence $\left|\left\langle u_{N}, g\right\rangle\right|=|\langle u, f\rangle|<\epsilon$. This shows that $u_{N} \in B_{\pi_{N}}^{0}(G / N)$, by Theorem $2.2(\mathrm{i})$.

Conversely, let $v \in B_{\pi_{N}}^{0}(G / N)$ and let $u=v \circ q \in B(G)$. Clearly, $u_{N}=v$. Given $\epsilon>0$, again by Theorem 2.2(i), there exists a compact subset $C$ of $G / N$ such that $|\langle v, g\rangle|<\epsilon$ for all $g \in L^{1}(G / N \backslash C)$ with $\left\|\pi_{N}(g)\right\| \leq 1$. Let $K=q^{-1}(C)$ 
and $f \in L^{1}(G \backslash K)$ be such that $\|\pi(f)\| \leq 1$. Then the function $h$ on $G / N$ defined by $h(x N)=\int_{N} f(x t) d t, x \in G$, belongs to $L^{1}(G / N \backslash C)$ and satisfies

$$
\left\langle\pi_{N}(h) \xi, \eta\right\rangle=\langle\pi(f) \xi, \eta\rangle
$$

for all $\xi, \eta \in P\left(\mathcal{H}_{\pi}\right)$. As before, this implies $\left\|\pi_{N}(h)\right\| \leq 1$, and, since $\langle v, h\rangle=\langle u, f\rangle$, it follows that $|\langle u, f\rangle|<\epsilon$. This proves that $u \in \phi\left(B_{\pi}^{0}(G)\right.$ ) (Theorem 2.2(i)).

The second statement of the lemma follows from the first and the fact that $\phi$ maps $A_{c}(G)$ onto $A_{c}(G / N)$.

Since $\phi$ maps $A(G)$ onto $A(G / N)$, Lemma 3.9 implies

Corollary 3.10. (i) If $\mathcal{B}_{\pi}^{0}(G)=A(G)$, then $\mathcal{B}_{\pi_{N}}^{0}(G / N)=A(G / N)$.

(ii) If $B_{\pi}^{0}(G)=A(G)$, then $B_{\pi_{N}}^{0}(G / N)=A(G / N)$.

\section{When does $\mathcal{B}_{\pi}^{0}(G)$ EQUal $A(G)$ ?}

As noticed earlier (see Lemma 3.1 and Corollary 2.5), if a representation $\pi$ of $G$ weakly contains the regular representation $\rho$, then $B_{\pi}^{0}(G)=A(G)=\mathcal{B}_{\pi}^{0}(G)$. Conversely, if $B_{\pi}^{0}(G)=A(G)$, then $A(G) \subseteq B_{\pi}(G)$, whence $\rho \prec \pi$. The question of whether the weaker hypothesis that

$$
A(G)=\mathcal{B}_{\pi}^{0}(G)=A_{c}(G)+\overline{B_{\pi}(G) \cap A_{c}(G)}
$$

already implies that $\rho \prec \pi$ appears to be very difficult. In this section we obtain a number of results (Theorem 4.2 and the corollaries of Theorem 4.9) which support the conjecture that this question has an affirmative answer for general locally compact groups.

Lemma 4.1. Let $\pi$ be a representation of the locally compact group $G$, and suppose that

$$
A_{c}(G)+\left(A(G) \cap B_{\pi}(G)\right)=A(G) .
$$

Then $\rho_{H} \prec \pi \mid H$ for every closed subgroup $H$ of $G$ such that $G / H$ is not compact.

Proof. We first assume that $G$ is $\sigma$-compact. Let $K_{n}, n \in \mathbb{N}$, be compact subsets of $G$ such that $\bigcup_{n=1}^{\infty} K_{n}=G$. Then, by hypothesis,

$$
\begin{gathered}
A(G)=\bigcup_{n=1}^{\infty} A_{K_{n}}(G)+\left(A(G) \cap B_{\pi}(G)\right) \\
=\bigcup_{n=1}^{\infty} \overline{A_{K_{n}}(G)+\left(A(G) \cap B_{\pi}(G)\right)} .
\end{gathered}
$$

By Baire's Category Theorem, at least one of these countably many closed subspaces of $A(G)$ must have non-void interior in $A(G)$. It follows that, for some compact subset $K$ of $G$,

$$
A(G)=\overline{A_{K}(G)+\left(A(G) \cap B_{\pi}(G)\right)} .
$$

Now let $H$ be a closed subgroup of $G$ such that $G / H$ is not compact. Then there exists $x \in G$ such that $H \cap x K=\emptyset$. Choose a representation $\sigma$ of $G$ such that $A_{\sigma}(G)=B_{\pi}(G)$. Since the restriction map from $B(G)$ to $B(H)$ is norm-continuous and

$$
A(G)=\overline{A_{x K}(G)+\left(A(G) \cap B_{\pi}(G)\right)},
$$


we conclude that

$$
\begin{gathered}
A(H)=A(G) \mid H \subseteq \overline{\left(A(G) \cap A_{\sigma}(G)\right) \mid H} \\
\subseteq A_{\sigma}(G) \mid H \subseteq A_{\sigma \mid H}(H) \subseteq B_{\pi \mid H}(H) .
\end{gathered}
$$

This implies that $\rho_{H}$ is weakly contained in $\pi \mid H$.

Now we drop the assumption that $G$ be $\sigma$-compact. However, to reduce to this case, observe first that if $F$ is any closed subgroup of $G$, then

$$
A(F)=A(G) \mid F \subseteq A_{c}(F)+\left(A(F) \cap B_{\pi \mid F}(F)\right) \subseteq A(F),
$$

and hence $\pi \mid F$ satisfies the original hypothesis as well. Let $\mathcal{F}$ denote the set of all $\sigma$-compact open subgroups of $G$. Let $q: G \rightarrow G / H$ denote the quotient map. Since $G / H$ is non-compact, there exists a $\sigma$-compact open subgroup $F_{0}$ of $G$ such that $q\left(F_{0}\right)$ fails to be compact. It follows that, for every $F \in \mathcal{F}$ with $F \supseteq F_{0}, q(F)$ is non-compact, and hence $F / F \cap H$ is not compact. For $\rho_{H} \prec \pi \mid H$ it suffices to show that $\rho_{H}|F \cap H \prec(\pi \mid H)| F \cap H$ for every $F \in \mathcal{F}, F \supseteq F_{0}$. Since $\rho_{H} \mid F \cap H \sim \rho_{F \cap H}$ and $(\pi \mid H)|F \cap H=\pi| F \cap H$, we have to verify that $\rho_{F \cap H} \prec \pi \mid F \cap H$ for all $F \in \mathcal{F}, F \supseteq F_{0}$. This follows now from the first part of the proof.

Theorem 4.2. Let $G$ be a locally compact group that fails to be compactly generated. Let $\pi$ be a representation of $G$ such that $\mathcal{B}_{\pi}^{0}(G)=A(G)$. Then $\rho_{G} \prec \pi$.

Proof. Let $\mathcal{H}$ denote the set of all compactly generated open subgroups of $G$. For any $H \in \mathcal{H}, G / H$ is non-compact since $G$ is not compactly generated. Hence $\rho_{H} \prec \pi \mid H$ by Lemma 4.1. Since $\rho_{G} \mid H \sim \rho_{H}$, it follows that $\rho_{G} \prec \pi$.

Although the next result will be improved later (Corollary 4.11) by using completely different methods, we present this special case in advance because it admits a much simpler proof.

Proposition 4.3. Let $G$ be an infinite discrete group and $\pi$ any representation of $G$. If $\mathcal{B}_{\pi}^{0}(G)=A(G)$, then $\pi$ weakly contains the regular representation.

Proof. By the same arguments as in the proof of Lemma 4.1, we can assume that $G$ is countable (and infinite). Then (compare the proof of Lemma 4.1) there exists a finite subset $F$ of $G$ such that

$$
A(G)=\overline{A_{F}(G)+\left(A(G) \cap B_{\pi}(G)\right.} .
$$

Let $I=\operatorname{ker} \pi$, so that $B_{\pi}(G)=\left(C^{*}(G) / I\right)^{*}$, and let $L=A_{F}(G)+B_{\pi}(G) \subseteq$ $B(G)$. Then $L\left|I=A_{F}(G)\right| I$, a finite-dimensional subspace of $I^{*}$. Now, $B_{\rho}(G)=$ $\overline{A(G)}^{w^{*}} \subseteq \bar{I}^{w^{*}}$. Since the map $u \rightarrow u \mid I$ from $B(G)$ onto $I^{*}$ is $w^{*}$-continuous, it follows that

$$
B_{\rho}(G)\left|I \subseteq \bar{L}^{w^{*}}\right| I \subseteq \overline{L \mid I}^{w^{*}}={\overline{A_{F}(G) \mid I}}^{w^{*}}=A_{F}(G) \mid I,
$$

since the latter space is finite dimensional.

Thus suppose that $\rho \mid I$ is nonzero. Then $\rho \mid I$ is a nonzero finite-dimensional representation. Let $J=I \cap \operatorname{ker} \rho$. Then $\rho$ is a finite-dimensional representation of $I / J$ and $I / J$ is an ideal of $\rho\left(C^{*}(G)\right)$. From this we conclude that $\rho$ has a finite-dimensional subrepresentation, which is impossible since $G$ is infinite. Thus $\rho$ annihilates $I$, whence $\rho \prec \pi$. 
For any function $u$ on $G$, let $u^{\vee}$ be defined by $u^{\vee}(x)=u\left(x^{-1}\right)$. Then $u \rightarrow u^{\vee}$ is a linear isometry of $B(G)$ onto $B(G)$ mapping $A(G)$ onto $A(G)$. For $T \in V N(G)$, let $T^{\vee} \in V N(G)$ be defined by $\left\langle u, T^{\vee}\right\rangle=\left\langle u^{\vee}, T\right\rangle, u \in A(G)$. Then $T \rightarrow T^{\vee}$ is an isometric anti-automorphism of $V N(G)$.

Note that, for $u \in A(G)=V N(G)_{*} \subseteq V N(G)^{*}$ and $T \in V N(G)$, the Sakai products $T \cdot u$ and $u \cdot T$ in $A(G)$ are given by

$$
\langle T \cdot u, S\rangle=\langle u, S T\rangle \text { and }\langle u \cdot T, S\rangle=\langle u, T S\rangle
$$

for $S \in V N(G)$, respectively. Indeed, this follows from [36 p. 123, Theorem 2.7]. Note also that $T \cdot u$ is different from the product $T u$ as defined in [15, (3.16)]. One easily verifies that $(T \cdot u)^{\vee}=u^{\vee} \cdot T^{\vee}$ and $(u \cdot T)^{\vee}=T^{\vee} \cdot u^{\vee}$. The following lemma is similar to [15, Proposition 3.17].

Lemma 4.4. If $u \in A(G) \cap L^{2}(G)$, then $\left(T \cdot u^{\vee}\right)^{\vee}=T(u)$ a.e. for every $T \in$ $V N(G)$.

Proof. Let $u \in A(G) \cap L^{2}(G)$ and $g \in C_{c}(G)$. Then the $w^{*}$-continuous linear functionals on $V N(G)$ given by

$$
T \rightarrow\left(T \cdot u^{\vee}\right)^{\vee}(\rho(g))=\int_{G}\left(T \cdot u^{\vee}\right)^{\vee}(x) g(x) d x
$$

and

$$
T \rightarrow \int_{G} T(u)(x) g(x) d x
$$

agree on operators of the form $T=\rho(y), y \in G$, and hence are equal. So $\left(T \cdot u^{\vee}\right)^{\vee}=$ $T(u)$ a.e..

Lemma 4.5. Let $\sigma$ be a representation of $G$ such that $A_{\sigma}(G) \subseteq L^{2}(G)$. Then the identity map is a continuous embedding of $A_{\sigma}(G)$ into $L^{2}(G)$.

Proof. Let $j: A_{\sigma}(G) \rightarrow L^{2}(G)$ be the identity map and let $\left(u_{n}\right)_{n}$ be a sequence in $A_{\sigma}(G)$ such that $u_{n} \rightarrow u$ in $A_{\sigma}(G)$ and $j\left(u_{n}\right) \rightarrow w$ in $L^{2}(G)$. Then there exists a subsequence $\left(u_{n_{k}}\right)_{k}$ such that $j\left(u_{n_{k}}\right)_{k} \rightarrow w$ pointwise a.e.. Since also $u_{n} \rightarrow u$ pointwise, it follows that $w=j(u)$. By the closed graph theorem, $j$ is continuous.

If $\sigma$ is a representation of $G$ such that $A_{\sigma}(G) \subseteq A(G)$, recall that there exists a unique central projection $P_{\sigma}$ in $V N(G)$ such that $A_{\sigma}(G)=P_{\sigma} \cdot A(G)$. Let $\mathcal{L}_{\sigma}=P_{\sigma}\left(L^{2}(G)\right)$. Let $\mathcal{L}_{\sigma}^{-}$denote the space of all complex conjugates of functions in $\mathcal{L}_{\sigma}$.

Lemma 4.6. Let $\sigma$ be a representation of $G$ such that $A_{\sigma}(G) \subseteq L^{2}(G)$. Then $B_{\sigma}(G) \subseteq{\overline{A_{\sigma}(G)}}^{\|\cdot\|_{2}}$ and $\overline{A_{\sigma}(G)} \|^{\|\cdot\|_{2}}=\mathcal{L}_{\sigma}^{-}$.

Proof. It is sufficient to show that every positive definite function $u$ in $B_{\sigma}(G)$ of norm one belongs to $\overline{A_{\sigma}(G)}\|\cdot\|_{2}$. There exists a net $\left(u_{\alpha}\right)_{\alpha}$ in $A_{\sigma}(G)$ of positive definite functions of norm one converging to $u$ uniformly on compact subsets of $G$ [11, Proposition 18.1.4]. Then

$$
\int_{G} f(x) u_{\alpha}(x) d x \rightarrow \int_{G} f(x) u(x) d x
$$


for all $f \in C_{c}(G)$. Since, by Lemma $4.5,\left(u_{\alpha}\right)_{\alpha}$ is bounded in the $\|\cdot\|_{2}$-norm, by $c$ say, it follows that

$$
\left|\int_{G} f(x) u(x) d x\right| \leq c\|f\|_{2}
$$

for all $f \in C_{c}(G)$. Thus $f \rightarrow \int_{G} f(x) u(x) d x$ extends to a bounded linear functional on $L^{2}(G)$, and this implies that $u \in L^{2}(G)$. However, since $u_{\alpha} \rightarrow u$ weakly and $\overline{A_{\sigma}(G)}\|\cdot\|_{2}$ is weakly closed, we even have $u \in{\overline{A_{\sigma}(G)}}^{\|\cdot\|_{2}}$.

By Lemma 4.4 we have

$$
\left(P_{\sigma} \cdot A_{c}(G)\right)^{\vee}=\left(P_{\sigma} \cdot A_{c}(G)^{\vee}\right)^{\vee}=P_{\sigma}\left(A_{c}(G)\right) \subseteq \mathcal{L}_{\sigma},
$$

and hence $A_{\sigma}(G)^{\vee}=\left(P_{\sigma} \cdot A(G)\right)^{\vee} \subseteq \mathcal{L}_{\sigma}$, since the embedding $j: A_{\sigma}(G) \rightarrow L^{2}(G)$ is continuous (Lemma 4.5). On the other hand, $A_{c}(G)$ is dense in $L^{2}(G)$, and therefore $P_{\sigma}\left(A_{c}(G)\right)$ is dense in $\mathcal{L}_{\sigma}$. It follows that

$$
\overline{A_{\sigma}(G)}\left\|^{\|\cdot\|_{2}}=\overline{\left(\left(A_{\sigma}(G)^{\vee}\right)^{-}\right)}\right\| \cdot \|_{2}=\mathcal{L}_{\sigma}^{-} .
$$

Proposition 4.7. Let $\sigma$ be a representation of $G$ such that $A_{\sigma}(G) \subseteq L^{2}(G)$. Then $A_{\sigma}(G)=B_{\sigma}(G)$.

Proof. There exists a representation $\tau$ of $G$ such that $B_{\sigma}(G)=A_{\tau}(G)$. By Lemma 4.6, ${\overline{A_{\tau}(G)}}^{\|\cdot\|_{2}}=\mathcal{L}_{\tau}^{-}$and

$$
\mathcal{L}_{\sigma}^{-}={\overline{A_{\sigma}(G)}}^{\|\cdot\|_{2}} \subseteq{\overline{B_{\sigma}(G)}}^{\|\cdot\|_{2}}={\overline{A_{\tau}(G)}}^{\|\cdot\|_{2}} \subseteq \mathcal{L}_{\sigma}^{-} .
$$

Thus $\mathcal{L}_{\sigma}=\mathcal{L}_{\tau}$, whence $P_{\sigma}=P_{\tau}$ and

$$
A_{\sigma}(G)=P_{\sigma} \cdot A(G)=P_{\tau} \cdot A(G)=A_{\tau}(G)=B_{\sigma}(G) .
$$

Theorem 4.8. Let $\sigma$ be a representation of $G$ such that $A_{\sigma}(G) \subseteq L^{2}(G)$. Then $G$ is unimodular, $\operatorname{supp} \sigma$ is discrete, and $\sigma$ decomposes into a direct sum of squareintegrable irreducible representations.

Proof. We first show that $\operatorname{supp} \sigma$ is discrete. For that we have to verify that every subset $T$ of $\operatorname{supp} \sigma$ is closed. Let $\sigma_{T}$ be the direct sum of all $\tau \in T$. Then, by Proposition 4.7,

$$
A_{\sigma_{T}}(G) \subseteq B_{\sigma_{T}}(G) \subseteq B_{\sigma}(G) \subseteq L^{2}(G) .
$$

Applying Proposition 4.7 again, we obtain that $B_{\sigma_{T}}(G)=A_{\sigma_{T}}(G)$. We claim that $\operatorname{supp} \sigma_{T}=T$. For that, let $\pi \in \operatorname{supp} \sigma_{T}$. Then $B_{\pi}(G) \subseteq B_{\sigma_{T}}(G)=A_{\sigma_{T}}(G)$. It follows that $\pi$ is equivalent to a subrepresentation of $\sigma_{T}$, whence $\pi=\tau$ for some $\tau \in T$, as required.

Notice next that if $\tau \in \operatorname{supp} \sigma$, then $A_{\tau}(G) \subseteq B_{\sigma}(G)$. Since $B_{\sigma}(G)=A_{\sigma}(G)$ by Proposition 4.7, we get that $\tau$ is a subrepresentation of $\sigma$, and then all coefficient functions of $\tau$ are in $L^{2}(G)$ since $A_{\tau}(G) \subseteq A_{\sigma}(G) \subseteq L^{2}(G)$. It follows now from [14, p. 215, remark after Theorem 3] that $G$ is unimodular.

Finally, let $\mathcal{L}$ denote the closed linear subspace of $\mathcal{H}_{\sigma}$ generated by all the irreducible subspaces of $\mathcal{H}_{\sigma}$, and let $\tau$ denote the restriction of $\sigma$ to the orthogonal complement of $\mathcal{L}$ in $\mathcal{H}_{\sigma}$. Then $\tau$ does not contain any irreducible subrepresentation. However, this contradicts $A_{\tau}(G) \subseteq L^{2}(G)$, by what we have seen in the preceding paragraph. Thus $\sigma$ decomposes into a direct sum of irreducible representations. 
Theorem 4.9. Let $G$ be a locally compact group and suppose that there exists a representation $\pi$ of $G$ such that $\mathcal{B}_{\pi}^{0}(G)=A(G)$ and nevertheless $\pi$ does not weakly contain the regular representation. Then there exists $\sigma \in \widehat{G}_{r}$ such that the singleton $\{\sigma\}$ is open in $\widehat{G}_{r}$ and $A_{\sigma}(G)=P_{\sigma} \cdot A_{c}(G)$.

Proof. There exists a representation $\pi_{0}$ such that $A_{\pi_{0}}(G)=\overline{B_{\pi}(G) \cap A_{c}(G)}$. Then, since $A_{\pi_{0}}(G) \subseteq A(G), \pi_{0}$ is quasi-equivalent to a subrepresentation of $\rho$. Moreover, $\pi_{0} \prec \pi$, but $\pi_{0}$ is not weakly equivalent to $\rho$. Let $Q=I-P_{\pi_{0}}$, a nonzero central projection in $V N(G)$, and let $\pi_{1}$ be a representation of $G$ such that $A_{\pi_{1}}(G)=$ $Q \cdot A(G)$. Then $Q=P_{\pi_{1}}$ and, by hypothesis and the definition of $\mathcal{B}_{\pi}^{0}(G)$,

$$
\begin{aligned}
A_{\pi_{1}}(G) & =Q \cdot A_{c}(G)+Q \cdot\left(P_{\pi_{0}} \cdot \overline{B_{\pi}(G) \cap A_{c}(G)}\right) \\
& =Q \cdot A_{c}(G)+\left(Q P_{\pi_{0}}\right) \cdot \overline{B_{\pi}(G) \cap A_{c}(G)}=Q \cdot A_{c}(G) .
\end{aligned}
$$

In particular, $A_{\pi_{1}}(G) \subseteq L^{2}(G)$, and hence supp $\pi_{1}$ is discrete by Theorem 4.8. Now

$$
A_{\pi_{0}}(G)+A_{\pi_{1}}(G)=P_{\pi_{0}} \cdot A(G)+\left(I-P_{\pi_{0}}\right) \cdot A(G)=A(G) .
$$

It follows that $\widehat{G}_{r}=\operatorname{supp} \pi_{0} \cup \operatorname{supp} \pi_{1}$ and $\operatorname{supp} \pi_{0} \neq \widehat{G}_{r}$. Let $\sigma$ be any point of $\widehat{G}_{r} \backslash \operatorname{supp} \pi_{0}$. Then $\{\sigma\}$ is open in $\widehat{G}_{r}$, since $\widehat{G}_{r} \backslash \operatorname{supp} \pi_{0}$ is discrete and open in $\widehat{G}_{r}$. Finally,

$$
A_{\sigma}(G)=P_{\sigma} \cdot A(G)=P_{\sigma} \cdot\left(P_{\sigma} \cdot A(G)\right) \subseteq P_{\sigma} \cdot\left(P_{\pi_{1}} \cdot A(G)\right)=P_{\sigma} \cdot A_{c}(G) .
$$

Thus $A_{\sigma}(G)=P_{\sigma} \cdot A_{c}(G)$, as was to be shown.

Corollary 4.10. Let $G$ be a locally compact group with non-compact centre. If $\pi$ is any representation of $G$ with $\mathcal{B}_{\pi}^{0}(G)=A(G)$, then $\pi$ weakly contains the regular representation.

Proof. Let $\pi$ be such that $\mathcal{B}_{\pi}^{0}(G)=A(G)$, and suppose that nevertheless $\pi$ does not weakly contain the regular representation $\rho$. Then, by Theorem 4.9 , there exists $\tau \in \widehat{G}_{r}$ so that the singleton $\{\tau\}$ is open in $\widehat{G}_{r}$.

Let $Z$ denote the centre of $G$, and recall that for any $\sigma \in \widehat{G}$ there is a unique character $\chi_{\sigma} \in \widehat{Z}$ such that $\sigma \mid Z$ is a multiple of $\chi_{\sigma}$. It suffices to show that $\left\{\chi_{\tau}\right\}$ is open in $\widehat{Z}$, because this contradicts the non-compactness of $Z$. Assuming that $\left\{\chi_{\tau}\right\}$ fails to be open, we get that the set $X=\left\{\chi \in \widehat{Z}: \chi \neq \chi_{\tau}\right\}$ is dense in $\widehat{Z}$. This implies that

$$
\rho \sim \operatorname{ind}_{Z}^{G}(\rho \mid Z) \sim\left\{\operatorname{ind}_{Z}^{G} \chi: \chi \in X\right\} .
$$

Since $\{\tau\}$ is open in $\widehat{G}_{r}=\operatorname{supp} \rho$, we conclude that $\tau \in \operatorname{supp}\left(\operatorname{ind}_{Z}^{G} \chi\right)$ for some $\chi \in X$, and this yields that $\chi_{\tau}=\chi$, a contradiction.

Corollary 4.11. Let $G$ be a non-compact SIN-group and let $\pi$ be a representation of $G$. Then $\mathcal{B}_{\pi}^{0}(G)=A(G)$ (if and) only if $\pi$ weakly contains the regular representation.

Proof. The statement is a consequence of Theorem 4.9, since the regular representation of a non-compact SIN-group cannot contain an irreducible subrepresentation. Indeed, this can be seen as follows. Suppose that $\pi$ is an irreducible subrepresentation of $\rho$. Then there is a central projection $E_{\pi}$ in $V N(G)$ such that $E_{\pi} V N(G)$ is isomorphic to the algebra of all bounded operators on $\mathcal{H}_{\pi}$. Since $V N(G)$ is a 
finite von Neumann algebra [11, Proposition 13.10.5], this forces $\pi$ to be finitedimensional. However, the regular representation of a non-compact group cannot have a finite-dimensional subrepresentation (see [26]).

It follows from a deep theorem of Harish-Chandra that many semisimple Lie groups, including all complex analytic ones, do not have a discrete series (compare [38, Chapter 10]). Thus Theorem 4.9 shows that, for any such group $G$ and any representation $\pi$ of $G, \mathcal{B}_{\pi}^{0}(G)=A(G)$ only if $\pi$ weakly contains the regular representation.

We have not been able to show that, for an arbitrary locally compact group $G$ and any representation $\pi$ of $G, \mathcal{B}_{\pi}^{0}(G)$ equals $A(G)$ only when $\pi$ weakly contains the regular representation. We hope, however, that in order to accomplish this the following proposition will prove useful.

Let $\mathcal{G}$ denote the collection of all locally compact groups $G$ with the property that if $\pi$ is any representation of $G$ such that $\mathcal{B}_{\pi}^{0}(G)=A(G)$, then $\rho \prec \pi$.

Proposition 4.12. $\mathcal{G}$ is closed under forming projective limits.

Proof. We first note that if $N$ is a compact normal subgroup of a locally compact group $G$ and $q: G \rightarrow G / N$ is the quotient homomorphism, then

$$
\widehat{G / N} \circ q \cap \widehat{G}_{r}=(\widehat{G / N})_{r} \circ q \text {. }
$$

Indeed, if $\tau \in(\widehat{G / N})_{r}$, then $\tau \circ q \prec \rho_{G / N} \circ q \leq \rho_{G}$. For the reverse inclusion, observe that if $\pi$ and $\sigma$ are representations of $G$ such that $\pi \prec \sigma$, then it is easily verified that $\pi_{N} \prec \sigma_{N}$. Thus, if $\tau \in \widehat{G / N}$ is such that $\tau \circ q \prec \rho$, then $\tau=(\tau \circ q)_{N} \prec \rho_{N}$. On the other hand, with $P$ as before Lemma 3.9, $P\left(L^{2}(G)\right)$ is canonically isomorphic to $L^{2}(G / N)$, and hence $\rho_{N}$ is equivalent to $\rho_{G / N}$. This shows that $\tau \circ q \prec \rho_{N} \circ q=$ $\rho_{G / N} \circ q$, as required.

Suppose now that $G$ is a projective limit of groups $G / N_{\alpha}, \alpha \in A$, where each $G / N_{\alpha}$ belongs to $\mathcal{G}$. Let $\pi$ be a representation of $G$ such that $\mathcal{B}_{\pi}^{0}(G)=A(G)$. Choose any $\xi \in \mathcal{H}_{\pi}, \xi \neq 0$. Then, by continuity, $\int_{N_{\alpha}} \pi(t) \xi d \mu_{\alpha}(t) \neq 0$ for $\alpha \geq \alpha_{0}$, where $\mu_{\alpha}$ is normalized Haar measure of $N_{\alpha}$. Thus, for $\alpha \geq \alpha_{0}, \pi \mid N_{\alpha}$ contains the trivial representation of $N_{\alpha}$, and therefore we can assume that $\pi_{N_{\alpha}}$ is defined for all $\alpha \in A$. By Corollary 3.10, for every $\alpha$,

$$
\mathcal{B}_{\pi_{N_{\alpha}}}^{0}\left(G / N_{\alpha}\right)=A\left(G / N_{\alpha}\right)
$$

whence $\rho_{G / N_{\alpha}} \prec \pi_{N_{\alpha}}$, since $G / N_{\alpha} \in \mathcal{G}$. Thus, with $q_{\alpha}: G \rightarrow G / N_{\alpha}$,

$$
\rho_{G / N_{\alpha}} \circ q_{\alpha} \prec \pi_{N_{\alpha}} \circ q_{\alpha},
$$

$\alpha \in A$. Since, for all $\xi, \eta \in \mathcal{H}_{\pi_{N_{\alpha}}} \subseteq \mathcal{H}_{\pi}$,

$$
\left\langle\pi_{N_{\alpha}}\left(x N_{\alpha}\right) \xi, \eta\right\rangle=\langle\pi(x) \xi, \eta\rangle,
$$

it follows that $\pi_{N_{\alpha}} \circ q_{\alpha}$ is a subrepresentation of $\pi$. Hence $\rho_{G / N_{\alpha}} \circ q_{\alpha} \prec \pi$ for every $\alpha$. Now, $\widehat{G}=\bigcup_{\alpha} \widehat{G / N_{\alpha}} \circ q_{\alpha}$ [31, Proposition 2.2], and hence, by what we have seen above,

$$
\widehat{G}_{r}=\bigcup_{\alpha}\left(\widehat{G / N_{\alpha}} \circ q_{\alpha} \cap \widehat{G}_{r}\right)=\bigcup_{\alpha}\left(\widehat{G / N_{\alpha}}\right)_{r} \circ q_{\alpha} .
$$

This proves that

$$
\rho \sim\left\{\rho_{G / N_{\alpha}} \circ q_{\alpha}: \alpha \in A\right\} \prec \pi
$$

as was to be shown. 


\section{A Dichotomy of $B_{\pi}^{0}(G)$ FOR SOME Classes of GROUPS}

Recall that whenever $G$ has a non-trivial compact normal subgroup $K$, then for $\pi=\operatorname{ind}_{K}^{G} 1_{K}$ we have $A_{c}(G) \subsetneq \mathcal{B}_{\pi}^{0}(G) \subsetneq A(G)$, and hence $\{0\} \subsetneq B_{\pi}^{0}(G) \subsetneq A(G)$ (see Lemma 3.8). However, when $G$ does not contain any non-trivial compact normal subgroup, it is not unlikely that, for an arbitrary representation $\pi$ of $G$, either $B_{\pi}^{0}(G)=\{0\}$ or $B_{\pi}^{0}(G)=A(G)$. At least, we are able to establish this somewhat surprising dichotomy for $B_{\pi}^{0}(G)$ for some classes of locally compact groups. Note that then the analogous dichotomy holds for $\mathcal{B}_{\pi}^{0}(G)$; that is, for any representation $\pi$ of $G$, either $\mathcal{B}_{\pi}^{0}(G)=A_{c}(G)$ or $\mathcal{B}_{\pi}^{0}(G)=A(G)$.

We start with abelian groups, followed by an application of the result to nonabelian groups.

A locally compact group $G$ is called compact-free if the identity is the only element of $G$ that generates a relatively compact subgroup.

Lemma 5.1. Let $G$ be a compact-free locally compact abelian group and let $f \in$ $L_{c}^{\infty}(G)$. If $f \neq 0$, then the Fourier transform $\widehat{f}$ of $f$ vanishes only on a set of measure zero in $\widehat{G}$.

Proof. Choose a compactly generated open subgroup $H$ of $G$ such that $f=0$ on $G \backslash H$. Since $H$ is compact-free, by the structure theorem for compactly generated abelian groups, $H=\mathbb{Z}^{k} \times \mathbb{R}^{l}$ for certain $k, l \in \mathbb{N}_{0}$. There are $m_{1}, \ldots, m_{n} \in \mathbb{Z}^{k}$ and $f_{1}, \ldots, f_{n} \in L_{c}^{\infty}\left(\mathbb{R}^{l}\right)$ such that

$$
f(m, x)=\sum_{j=1}^{n} \delta_{m_{j}}(m) f_{j}(x)
$$

for all $x \in \mathbb{R}^{l}$ and $m \in \mathbb{Z}^{k}$. Thus

$$
\widehat{f \mid H}(z, y)=\sum_{j=1}^{n} z^{m_{j}} \widehat{f}_{j}(y)
$$

for all $z \in \mathbb{T}^{k}$ and $y \in \mathbb{R}^{l}$. Of course, we have used here the notation $z^{q}=z_{1}^{q_{1}} \ldots \cdot z_{k}^{q_{k}}$ for $z=\left(z_{1}, \ldots, z_{k}\right) \in \mathbb{T}^{k}$ and $q=\left(q_{1}, \ldots, q_{k}\right) \in \mathbb{Z}^{k}$. Since for suitably chosen $q \in \mathbb{Z}^{k}$, the function

$$
(z, y) \rightarrow z^{q} \sum_{j=1}^{n} z^{m_{j}} \widehat{f}_{j}(y)
$$

on $\mathbb{T}^{k} \times \mathbb{R}^{l}$ is analytic and $f \neq 0$, it follows that $\widehat{f \mid H}$ vanishes only on a set $\Gamma$ of measure zero in $\widehat{H}$.

Now, let $r: \widehat{G} \rightarrow \widehat{H}$ denote the restriction homomorphism $\alpha \rightarrow \alpha \mid H$. Since $f=0$ on $G \backslash H$, we have $\widehat{f}(\alpha)=\widehat{f \mid H}(r(\alpha))$ for each $\alpha \in \widehat{G}$. Hence $\widehat{f}$ vanishes only on $r^{-1}(\Gamma)$. It remains to verify that $r^{-1}(\Gamma)$ has measure zero. To that end, notice that since $\widehat{G / H}$ is compact, the continuous surjective homomorphism $r: \widehat{G} \rightarrow \widehat{H}$ is proper, and hence transfers Haar measure of $\widehat{G}$ to Haar measure of $\widehat{H}$. Thus $r^{-1}(\Gamma)$ is a null set.

In the sequel we shall say that the dichotomy holds for a locally compact group $G$ if for every unitary representation $\pi$ of $G$, either $B_{\pi}^{0}(G)=\{0\}$ or $B_{\pi}^{0}(G)=A(G)$.

Corollary 5.2. Let $G$ be a compact-free locally compact abelian group. Then the dichotomy holds for $G$. 
Proof. Suppose there exists a nonzero $u \in B_{\pi}(G) \cap A_{c}(G)$. We have to show that $\pi \sim \widehat{G}$. Recall that $u$ is a coordinate function of some representation $\sigma$ of $G$ which is weakly contained in $\pi$. Thus $u(x)=\langle\sigma(x) \xi, \eta\rangle$ for all $x \in G$, where $\xi, \eta \in \mathcal{H}_{\sigma}$. Then, for all $g \in L^{1}(G)$ and $a \in G$,

$$
(u * g)(a)=\int_{G} g(x)\langle\sigma(a) \xi, \sigma(x) \eta\rangle d x=\langle\sigma(a) \xi, \sigma(\bar{g}) \eta\rangle .
$$

Assume that $\operatorname{supp} \sigma \neq \widehat{G}$. Then there exists $g \in L^{1}(G)$ such that $g \neq 0$ and $\widehat{\bar{g}}=0$ on $\operatorname{supp} \sigma$. It follows from the above formula that $(u * g)(a)=0$ for all $a \in G$, and therefore $\widehat{u} \widehat{g}=0$ on $\widehat{G}$. However, $\widehat{g} \neq 0$ on some nonempty open subset of $\widehat{G}$, whereas $\widehat{u}=0$ only on a set of measure zero by Lemma 5.1. This contradiction shows that $\operatorname{supp} \sigma=\widehat{G}$, whence $\operatorname{supp} \pi=\widehat{G}$, as required.

Let $N$ be a closed normal subgroup of a locally compact group $G$. The action of $G$ on $N$ by inner automorphisms gives rise to an action of $G$ on representations of $N$ as follows. For $x \in G$ and any representation $\tau$ of $N$, let $x \cdot \tau$ be defined by $x \cdot \tau(y)=\tau\left(x^{-1} y x\right)$ for all $y \in N$. Let $\operatorname{Prim}(N)$ denote the primitive ideal space of $C^{*}(N)$, that is, the set of all kernels $\operatorname{ker} \tau, \tau \in \widehat{N}$, in $C^{*}(N)$, endowed with the hull-kernel topology. Then the above action induces actions $(x, \tau) \rightarrow x \cdot \tau$ and $(x, P) \rightarrow x \cdot P$ of $G$ on $\widehat{N}$ and on $\operatorname{Prim}(N)$, respectively.

Recall that $N$ is said to be regularly embedded in $G$ if the orbit space $\operatorname{Prim}(N) / G$ with the quotient topology is almost Hausdorff (that is, every nonempty closed subset contains a nonempty open Hausdorff subset) (see [35, Definitions 7.1 and $7.2])$.

Proposition 5.3. Let $G$ be a second countable amenable locally compact group and $N$ a closed normal subgroup of $G$ such that $N$ is regularly embedded in $G$. Suppose there exists a $G$-invariant dense open subset $V$ of $\widehat{N}$ such that for every $\sigma \in \widehat{G}$, either $\sigma \sim \operatorname{ind}_{N}^{G}(\sigma \mid N)$ or $\sigma \mid N \prec \widehat{N} \backslash V$. If $N$ satisfies the dichotomy, then so does $G$.

Proof. Suppose that the dichotomy holds for $N$ and let $\pi$ be a representation of $G$ such that $B_{\pi}^{0}(G) \neq\{0\}$. Then $B_{\pi \mid N}^{0}(N) \neq\{0\}$, and hence $B_{\pi}^{0}(N)=A(N)$. Since $N$ is amenable, it follows that $B_{\pi \mid N}(N)=B(N)$, and therefore $\pi \mid N \sim \widehat{N}$. Let

$$
S=\left\{\sigma \in \operatorname{supp} \pi: \sigma \sim \operatorname{ind}_{N}^{G}(\sigma \mid N)\right\} .
$$

Then, by hypothesis,

$$
\widehat{N} \sim \pi \mid N \sim\{\sigma \mid N: \sigma \in \operatorname{supp} \pi\} \sim\{\sigma \mid N: \sigma \in S\} \cup\{\sigma|N: \sigma| N \prec \widehat{N} \backslash V\} .
$$

Since $V$ is dense in $\widehat{N}$, we get that $\widehat{N} \sim\{\sigma \mid N: \sigma \in S\}$. Now, since $G$ is second countable and $N$ is regularly embedded in $G$, for every $\sigma \in \widehat{G}$ there exists $\tau_{\sigma} \in \widehat{N}$ such that $\sigma \mid N \sim G\left(\tau_{\sigma}\right)$, the $G$-orbit of $\tau_{\sigma}$ in $\widehat{N}$ 35, Proposition 8.1].

Thus, given any $\sigma \in \widehat{G}$, there exist $\sigma_{n} \in S$ and $x_{n} \in G, n \in \mathbb{N}$, such that $x_{n} \cdot \tau_{\sigma_{n}} \rightarrow \tau_{\sigma}$ in $\widehat{N}$. Since inducing is continuous in Fell's subgroup representation topology [16, Theorem 4.2] and $\operatorname{ind}_{N}^{G}(x \cdot \tau)=\operatorname{ind}_{N}^{G} \tau$ for any representation $\tau$ of $N$ and $x \in G$, it follows that

$$
\sigma_{n} \sim \operatorname{ind}_{N}^{G}\left(x_{n} \cdot \tau_{\sigma_{n}}\right) \rightarrow \operatorname{ind}_{N}^{G} \tau_{\sigma} .
$$


Since $G$ is amenable, $\sigma \prec \operatorname{ind}_{N}^{G} \tau_{\sigma}$. It follows that $\sigma_{n} \rightarrow \sigma$ in $\widehat{G}$, whence $\sigma \in \bar{S} \subseteq$ $\operatorname{supp} \pi$. Since $\sigma$ is arbitrary, $\operatorname{supp} \pi=\widehat{G}$, and hence $B_{\pi}^{0}(G)=\overline{B(G) \cap A_{c}(G)}=$ $A(G)$.

There are numerous examples of locally compact groups satisfying the hypotheses of Proposition 5.3. For simplicity, we only present examples where the normal subgroup $N$ in Proposition 5.3 is abelian.

Example 5.4. (1) Let $G$ be the ( $a x+b)$-group, that is, the semidirect product $\mathbb{R}^{+} \ltimes$ $\mathbb{R}$, where $\mathbb{R}^{+}$acts on $\mathbb{R}$ by multiplication. Then $\widehat{G}=\widehat{\mathbb{R}^{+}} \cup\left\{\pi_{+}, \pi_{-}\right\}$, where $\pi_{+}$and $\pi_{-}$are induced from the characters of $\mathbb{R}$ corresponding to 1 and -1 , respectively. Since neither $\left\{\pi_{+}\right\}$nor $\left\{\pi_{-}\right\}$is dense in $\widehat{G}$, it follows from Proposition 5.3 that $B_{\pi}^{0}(G)=\{0\}$ for every irreducible representation $\pi$ of $G$. Note that this does not follow from any of the results of Section 3.

(2) The hypotheses of Proposition 5.3 are also fulfilled by $S O(2) \ltimes \mathbb{R}^{2}$, the motion group of the plane, by the semi-direct product $\mathbb{Z} \ltimes \mathbb{R}$, where $n \in \mathbb{Z}$ acts on the reals by multiplication with $e^{n}$ and by the semidirect product of $\mathbb{Z}_{2}$ with $\mathbb{Z}$. Note that these examples show that the dichotomy may well hold when $G$ fails to be compactfree.

(3) Another class of examples is provided by nilpotent groups of the form $G=$ $\mathbb{R} \ltimes \mathbb{R}^{n}$. Here every irreducible representation either is a character of $G$ or is induced from some character of $\mathbb{R}^{n}$. In particular, this includes the Heisenberg group over $\mathbb{R}$.

(4) Let $H$ be a closed subgroup of $G L(n, \mathbb{R})$, and form the semi-direct product $G=H \ltimes \mathbb{R}^{n}$ defined by the natural action of $H$ on $\mathbb{R}^{n}$. Suppose there exists an open free $H$-orbit $U$ in $\widehat{\mathbb{R}^{n}}=\mathbb{R}^{n}$, where free means that the map $h \rightarrow h \cdot \chi$ from $H$ onto $U, \chi \in U$, is injective. Note that the existence of such an orbit forces $H$ to be $n$-dimensional as a Lie group. Then the union $V$ of all free $H$-orbits is dense in $\widehat{\mathbb{R}^{n}}$. Then $G$ satisfies the hypothesis of Proposition 5.3. Indeed, the freeness condition implies that $\operatorname{ind}_{\mathbb{R}^{n}}^{G} \chi$ is irreducible for every $\chi \in V$, and since the orbit of $\chi$ is open, it follows from Mackey's theory that $\operatorname{ind}_{\mathbb{R}^{n}}^{G} \chi$ is the only irreducible representation $\sigma$ of $G$ such that $\sigma \mid \mathbb{R}^{n} \sim U$. Several 2-dimensional examples have been given in [6].

Let $f \in A_{c}(G)$. We shall use the following terminology. We say that $f$ generates $A(G)$ if the linear span of all two-sided translates of $f$ is norm dense in $A(G)$ and that $\rho(f)$ generates $V N(G)$ if the $w^{*}$-closed ideal of $V N(G)$ generated by $\rho(f)$ equals $V N(G)$.

Lemma 5.5. Let $f \in A_{c}(G)$. Then $f$ generates $A(G)$ if and only if $\rho(f)$ generates $V N(G)$.

Proof. For $u \in A_{c}(G)$, let $A(u)$ denote the norm-closed two-sided translationinvariant subspace of $A(G)$ generated by $u$, and $I(u)$ the smallest $w^{*}$-closed ideal of $V N(G)$ containing $\rho(u)$. We claim that an element $R \in V N(G)=A(G)^{*}$ annihilates $A(u)$ if and only if $R I\left(u^{\vee}\right)=\{0\}$.

For any $S, T \in V N(G)$ we have

$$
\langle S \cdot u \cdot T, R\rangle=\langle u \cdot T, R S\rangle=\langle u, T R S\rangle=\langle(R S) \cdot u, T\rangle .
$$

Since for closed subspaces of $A(G)$ translation invariance is the same as invariance, it follows that $R$ annihilates $A(u)$ precisely when $(R S) \cdot u=0$ for all $S \in V N(G)$. 
By Lemma 4.4, $(R S) \cdot u=0$ is equivalent to $R S\left(u^{\vee}\right)=0$ in $L^{2}(G)$. Since $R S\left(u^{\vee}\right)$ belongs to the left Hilbert algebra of $G$, we have

$$
\rho\left(R S\left(u^{\vee}\right)\right)=R S \rho\left(u^{\vee}\right)
$$

[12, p. 82, Proposition 3]. This in turn implies that $R$ annihilates $A(u)$ if and only if $R I\left(u^{\vee}\right)=\{0\}$.

Finally, since $f$ generates $A(G)$ if and only if $f^{\vee}$ generates $A(G)$, taking $u=f^{\vee}$ yields the statement of the lemma.

Theorem 5.6. Let $G$ be a non-compact SIN-group. Then the following two conditions are equivalent.

(i) The dichotomy holds for $G$.

(ii) $G$ has no non-trivial compact normal subgroup.

Proof. According to Lemma 3.8, we only have to show that (ii) implies (i).

Let $G_{F}$ denote the subgroup of $G$ consisting of all elements of $G$ with relatively compact conjugacy classes, and let $G_{F}^{c}$ be the set of all compact elements of $G_{F}$. Then $G_{F}$ is an open normal subgroup of $G$, and by the structure theory of SINgroups, $G_{F}^{c}$ is a closed normal subgroup of $G$ and $G_{F} / G_{F}^{c}$ is compact-free and abelian [21, Theorem 3.16, (1) and (3)]. Moreover, every element of $G_{F}^{c}$ is contained in some compact normal subgroup of $G[21$, Theorem 3.16, (2)]. Thus, by hypothesis (ii), $G_{F}^{c}$ is trivial, and hence $G_{F}$ is compact-free abelian.

Since $G$ is an SIN-group, there exists a unique faithful normal centre-valued trace $T \rightarrow T^{\sharp}$ on $V N(G)$ [21, p. 281, Theorem 3]. Let $E$ denote the closed subspace of $L^{2}(G)$ consisting of all central elements, that is, functions $g \in L^{2}(G)$ such that, for each $x \in G, g(y)=g\left(x^{-1} y x\right)$ for almost all $y \in G$. Let $P$ be the orthogonal projection of $L^{2}(G)$ onto $E$. Then $\rho(u)^{\sharp}=\rho(P u)$ for all $u \in C_{c}(G)$ (see [12, p. 282, Exercise $2 \mathrm{~b})]$ ).

We need a description of $P$. Since $G$ is an SIN-group, the inner automorphisms $i_{y}: G_{F} \rightarrow G_{F}, x \rightarrow y^{-1} x y$ of $G_{F}, y \in G$, form a relatively compact subgroup of the group $\operatorname{Aut}\left(G_{F}\right)$ of all topological automorphisms of $G_{F}$ 21. Let $\Gamma$ denote the closure of $\left\{i_{y}: y \in G\right\}$ in Aut $\left(G_{F}\right)$, and $d \gamma$ normalized Haar measure on $\Gamma$. Then, for $u \in C_{c}(G), P u$ is given by $P u(x)=0$ for $x \in G \backslash G_{F}$ and

$$
P u(x)=\int_{\Gamma} u(\gamma(x)) d \gamma
$$

for $x \in G_{F}$ (see [32]). Then $P u \in C_{c}(G)$, and if $u$ is positive definite, then so is $P u$.

Now, let $\pi$ be a unitary representation of $G$ such that $B_{\pi}(G) \cap A_{c}(G) \neq\{0\}$, and choose a nonzero function $f$ in $B_{\pi}(G) \cap A_{c}(G)$. It suffices to prove that $f$ generates $A(G)$. Equivalently, by Lemma 5.5 , it is enough to show that the operator $\rho(f)$ generates $V N(G)$. Note that $\rho(f)$ generates $V N(G)$ if (and only if) $I(f)^{\sharp}$ generates the centre $\mathcal{Z}(G)$ of $V N(G)$. Since $\left(\rho(f)^{*} \rho(f)\right)^{\sharp} \in I(f)^{\sharp}$, it suffices to prove that $\left(\rho(f)^{*} \rho(f)\right)^{\sharp}=\rho\left(f^{*} * f\right)^{\sharp}$ generates $\mathcal{Z}(G)$. For that, set $u=f^{*} * f \in A_{c}(G)$.

It follows from the above that $P u \neq 0, P u \in A_{c}(G)$ and $P u=0$ outside of $G_{F}$. In particular, $v=u \mid G_{F} \in A_{c}\left(G_{F}\right), v \neq 0$, and since $G_{F}$ is compact-free and abelian, $v$ generates $A\left(G_{F}\right)$ (Corollary 5.2). Thus $\rho(v)$ generates $V N\left(G_{F}\right)$, and since $P u$ vanishes on $G \backslash G_{F}$, this implies that $\rho(u)^{\sharp}=\rho(P u)$ generates $\mathcal{Z}(G)$. 


\section{Nilpotent GROUPS}

In this final section we add some more detailed results on nilpotent locally compact groups. We start with a lemma and a corollary which are of independent interest.

Lemma 6.1. Let $Z$ be a compact subgroup of the locally compact group $G$ that is contained in the centre of $G$, and let $\chi$ be a character of $Z$ and $\tau=\operatorname{ind}_{Z}^{G} \chi$. Then

$$
A_{\tau}(G)=\{u \in A(G): u(x z)=\chi(z) u(x) \text { for all } x \in G \text { and } z \in Z\} .
$$

Proof. Let $L$ denote the set of functions on the right-hand side. For $A_{\tau}(G) \subseteq L$ it suffices to show that every coordinate function of $\tau$ belongs to $L$. Let $u(x)=$ $\langle\tau(x) \xi, \eta\rangle$, where $\xi, \eta \in \mathcal{H}_{\tau}$. Then, since $\tau \mid Z$ is a multiple of $\chi$,

$$
u(x z)=\langle\tau(z) \tau(x) \xi, \eta\rangle=\chi(z)\langle\tau(x) \xi, \eta\rangle=\chi(z) u(x)
$$

for all $x \in G$ and $z \in Z$. Since $\tau$ is a subrepresentation of $\rho, A_{\tau}(G) \subseteq A(G)$, and hence $u \in L$.

For the converse, note first that $\rho$ is the direct sum of representations $\tau_{\lambda}=$ $\operatorname{ind}_{Z}^{G} \lambda, \lambda \in \widehat{Z}$, and that the representations $\tau_{\lambda}$ are pairwise disjoint. Therefore, by 1. (3.13) Corollaire 1], every $u \in A(G)$ has a unique decomposition $u=\sum_{\lambda \in \widehat{Z}} u_{\lambda}$, where $u_{\lambda} \in A_{\tau_{\lambda}}(G)$. Now, for all $x \in G$ and $z \in Z$,

$$
\sum_{\lambda} R_{z} u_{\lambda}(x)=u(x z)=\chi(z) u(x)=\sum_{\lambda} \chi(z) u_{\lambda}(x)
$$

This implies that $R_{z} u_{\lambda}=\chi(z) u_{\lambda}$ for all $\lambda \in \widehat{Z}$ and $z \in Z$. On the other hand, by the first part of the proof, $v(x z)=\lambda(z) v(x)$ for all $v \in A_{\tau_{\lambda}}(G), x \in G, z \in Z$. It follows that $u_{\lambda}=0$ for $\lambda \neq \chi$, and hence $u=u_{\chi} \in A_{\tau}(G)$.

Corollary 6.2. Let $G, Z, \chi$ and $\tau$ be as in Lemma 6.1. Then

$$
B_{\tau}(G)=\left\{u \in B_{\rho}(G): u(x z)=\chi(z) u(x) \text { for all } x \in G \text { and } z \in Z\right\} .
$$

Proof. Let $L$ denote the set of functions on the right. Since $L$ is $w^{*}$-closed in $B(G)$, it follows from Lemma 6.1 that $B_{\tau}(G) \subseteq L$.

Conversely, let $u \in L$ with $\|u\|=1$ be given. There exists a net $\left(v_{\alpha}\right)_{\alpha}$ in $A(G)$ such that $v_{\alpha} \rightarrow u$ in the $w^{*}$-topology of $B(G)$ and $\left\|v_{\alpha}\right\| \leq 1$ for all $\alpha$. Then $\left\|v_{\alpha}\right\| \rightarrow\|u\|=1$, and hence, replacing $v_{\alpha}$ by $\left\|v_{\alpha}\right\|^{-1} v_{\alpha}$, we can assume that $\left\|v_{\alpha}\right\|=1$ for all $\alpha$. Let $d t$ be normalized Haar measure on $Z$ and define $u_{\alpha}: G \rightarrow \mathbb{C}$ by

$$
u_{\alpha}(x)=\int_{Z} v_{\alpha}(x t) \overline{\chi(t)} d t=\int_{Z} \overline{\chi(t)} R_{t} v_{\alpha}(x) d t,
$$

where $R_{t}$ denotes (right) translation by $t \in Z$. Then $u_{\alpha} \in A(G)$ and $\left\|u_{\alpha}\right\| \leq$ $\int_{Z}\left\|R_{t} v_{\alpha}\right\| d t=1$ for every $\alpha$. Moreover, it is easily verified that $u_{\alpha}(x z)=\chi(z) u_{\alpha}(x)$ for all $x \in G$ and $z \in Z$. Thus $u_{\alpha} \in A_{\tau}(G)$ by Lemma 6.1. We claim that $u_{\alpha} \rightarrow u$ in the $w^{*}$-topology. Since $\left(u_{\alpha}\right)_{\alpha}$ is bounded in $B(G)$, it suffices to show that $\left\langle u_{\alpha}, f\right\rangle \rightarrow\langle u, f\rangle$ for each $f \in C_{c}(G)$. With $S=\operatorname{supp} f$, we have

$$
\begin{aligned}
& \left\langle u_{\alpha}, f\right\rangle-\langle u, f\rangle=\int_{G} f(x)\left(u_{\alpha}(x)-u(x)\right) d x \\
& =\int_{G} f(x) \int_{Z} \overline{\chi(t)}\left(R_{t} v_{\alpha}(x)-R_{t} u(x)\right) d t d x
\end{aligned}
$$




$$
=\int_{S Z} \int_{Z}\left(v_{\alpha}(x)-u(x) \overline{\chi(t)} f\left(x t^{-1}\right) d t d x .\right.
$$

Now, on the surface of the unit ball of $B(G)$ the $w^{*}$-topology coincides with the topology of uniform convergence on compact subsets of $G$ (see [19]). It follows that $\left\langle u_{\alpha}, f\right\rangle \rightarrow\langle u, f\rangle$, as required.

In the sequel, for any representation $\pi$, we continue to denote by $K_{\pi}$ and $P K_{\pi}$ the kernel and the projective kernel of $\pi$, respectively.

Theorem 6.3. Let $G$ be a non-compact connected nilpotent group, and let $\pi$ be an irreducible representation of $G$. Then the following conditions are equivalent.

(i) $B_{\pi}^{0}(G) \neq\{0\}$.

(ii) The centre of $G, Z(G)$, is compact, and $\pi \sim \operatorname{ind}_{Z(G)}^{G}(\pi \mid Z(G))$.

Proof. We first recall the well-known fact that if $H$ is a connected nilpotent group, then every compact normal subgroup of $H$ is contained in the centre of $H$.

Suppose now that (i) holds. Then, since $G$ is noncompact and the singleton $\{\pi\}$ is closed in $\widehat{G}, \pi$ is square integrable by Lemma 3.6 , and hence, in particular, $P K_{\pi}$ is compact. Thus, by the above, $P K_{\pi}=Z(G)$. Note that $B_{\pi}^{0}(G) \neq\{0\}$ if and only if $B_{\pi}(G) \cap A_{c}(G) \neq\{0\}$, and that functions in $B_{\pi}(G)$ are constant on cosets of $K_{\pi}$. Therefore, after passing to $G / K_{\pi}$, we can assume that $K_{\pi}=\{e\}$, that is, $\pi$ is a faithful representation of $G$, and $Z(G)$ is compact. Since $G$ is a projective limit of Lie groups and $\pi$ is a faithful irreducible representation of $G, G$ must be a Lie group [2, Proposition 2.2]. Let $H$ denote the simply connected covering group of $G$ and $q: H \rightarrow G$ the quotient homomorphism. Then $\pi \circ q \in \widehat{H}$ is square integrable modulo its kernel, which equals ker $q$. Since $G / Z(G)$ is simply connected, $Z(H)=q^{-1}(Z(G))$ is the smallest connected subgroup of $H$ containing ker $q$. Then $\operatorname{ind}_{Z(H)}^{H}(\pi \circ q \mid Z(H))$ is a multiple of $\pi \circ q$. This implies that $\operatorname{ind}_{Z(G)}^{G}(\pi \mid Z(G))$ is a multiple of $\pi$.

Conversely, let (ii) hold and let $\tau=\operatorname{ind}_{Z(G)}^{G} \chi$. Then $B_{\pi}(G)=B_{\tau}(G)$ and, by Lemma 6.1,

$$
B_{\tau}(G) \cap A_{c}(G)=\left\{u \in A_{c}(G): u(x z)=\chi(z) u(x) \text { for all } x \in G \text { and } z \in Z\right\} .
$$

Choose some $v \in A_{c}(G), v \neq 0$, and let $\varphi$ be a nonzero coordinate function of $\pi$. Then, since $\pi$ is irreducible and $\pi \mid Z(G) \sim \chi$, we have $\varphi(x z)=\chi(z) \varphi(x)$ for all $x \in G$ and $z \in Z(G)$. Let $u \in A_{c}(G)$ be defined by $u(x)=\varphi(x) v(x Z(G)), x \in G$. Then, for $x \in G$ and $z \in Z(G)$,

$$
u(x z)=\varphi(x z) v(x Z(G))=\chi(z) \varphi(x) v(x Z(G))=\chi(z) u(x) .
$$

This proves that $B_{\pi}(G) \cap A_{c}(G) \neq\{0\}$.

We remark that condition (ii) of Theorem 6.3 is known to be equivalent to the square integrability of $\pi$ [10, Section 4.5].

Corollary 6.4. Let $G$ be a non-compact connected nilpotent locally compact group. Consider the following two conditions.

(i) $B_{\pi}^{0}(G) \neq\{0\}$ for some $\pi \in \widehat{G}$.

(ii) $Z(G)$ is compact, and there exists a closed subgroup $K$ of $Z(G)$ such that $Z(G / K)=Z(G) / K$ and $Z(G) / K=\mathbb{T}$.

Then (i) $\Rightarrow$ (ii), and conversely (ii) $\Rightarrow$ (i) whenever $G$ is 2-step nilpotent. 
Proof. Suppose there exists an irreducible representation $\pi$ of $G$ such that $B_{\pi}^{0}(G)$ $\neq\{0\}$. Then, by Theorem 6.3,Z(G) is compact and $\pi \sim \operatorname{ind}_{Z(G)}^{G} \chi$ for some

$\chi \in \widehat{Z(G)}$. Let $K=K_{\pi}$, which equals $K_{\chi}$. Of course, $\chi$ is non-trivial, and hence $Z(G) / K=\mathbb{T}$, since $Z(G)$ is connected. Let $x \in G$ be such that $x K \in Z(G / K)$, and let $H$ denote the closed subgroup of $G$ generated by $Z(G)$ and $x$. Then, since $\pi$ can be viewed as an irreducible representation of $G / K, \pi \mid H$ is weakly equivalent to some character of $H$. This implies that $H=Z(G)$, whence $Z(G / K)=Z(G) / K$.

Conversely, if $K$ as in (ii) exists, then, after passing to $G / K$, we can assume that $Z(G)=\mathbb{T}$. Let $\chi(z)=z$ for $z \in \mathbb{T}$, and choose $\pi \in \widehat{G}$ such that $\pi \mid Z(G) \sim \chi$. If, in addition, $G$ is 2-step nilpotent, then $\pi \sim \operatorname{ind}_{H}^{G} \varphi$, where $H$ is a closed subgroup of $G$ containing $Z(G)$ and $\varphi$ is a $G$-invariant character of $H$. In fact, using an idea of 24, this has been shown in [25] for arbitrary 2-step nilpotent locally compact groups. We have to show that $H=Z(G)$. To see this, let $x \in H$. Then, for arbitrary $y \in G$, since $[y, x] \in Z(G)$,

$$
\pi(x)=\varphi(x) \operatorname{Id}_{\mathcal{H}_{\pi}}=\pi(y) \pi(x) \pi\left(y^{-1}\right)=\pi([y, x]) \pi(x)=\chi([y, x]) \pi(x) .
$$

Thus $\chi([G, x])=\{1\}$, and since $\chi$ is faithful, this shows that $x \in Z(G)$.

The implication (ii) $\Rightarrow$ (i) of Corollary 6.4 does not remain true for arbitrary connected nilpotent Lie groups. For instance, if $H$ denotes the (unique) 4-dimensional, 3-step nilpotent simply connected Lie group and $G$ the quotient of $H$ modulo a central integer subgroup, then $G$ satisfies (ii), whereas (i) fails. However, the condition that $G$ be 2-step nilpotent can be replaced with the weaker condition that every irreducible representation of $G$ is weakly equivalent to a representation induced by a $G$-invariant character of some closed normal subgroup of $G$.

Proposition 6.5. Let $G$ be a non-compact 2-step nilpotent locally compact group, and $\pi$ an irreducible representation of $G$. Then $B_{\pi}^{0}(G) \neq\{0\}$ if (and only if) $P K_{\pi}$ is compact.

Proof. By Lemma 3.3, for any representation $\pi$ of $G, B_{\pi}^{0}(G) \neq\{0\}$ implies that $P K_{\pi}$ is compact.

Now let $\pi$ be irreducible and suppose that $P K_{\pi}$ is compact. Since $G$ is 2-step nilpotent, there exist a closed normal subgroup $N$ of $G$ and a $G$-invariant character $\chi$ of $N$ such that $\pi \sim \operatorname{ind}_{N}^{G} \chi$. Obviously, then $N$ equals $P K_{\pi}$. The character $\chi$ extends to some $\varphi \in P(G)$ such that $\varphi \mid N=\chi$. Multiplying $\varphi$ with the pullback of some $\psi \in P(G / N) \cap C_{c}(G / N)$, we can assume that $\varphi \in C_{c}(G)$. Let $\pi_{\varphi}$ denote the representation associated to $\varphi$ by means of the Gelfand-Naimark-Segal construction. Then, since $\pi_{\varphi} \mid N$ is a multiple of $\chi$ and $G$ is amenable,

$$
\pi_{\varphi} \prec \operatorname{ind}_{N}^{G}\left(\pi_{\varphi} \mid N\right) \sim \operatorname{ind}_{N}^{G} \chi \sim \pi .
$$

It follows that $\varphi \in B_{\pi_{\varphi}}(G) \subseteq B_{\pi}(G)$. Thus $\varphi \in B_{\pi}(G) \cap C_{c}(G)$, and hence $B_{\pi}^{0}(G) \neq\{0\}$.

\section{REFERENCES}

[1] G. Arsac, Sur l'espace de Banach engendré par les coefficients d'une représentation unitaire, Publ. Dép. Math. (Lyon) 13 (1976), 1-101. MR 56:3188

[2] L. Baggett and K. F. Taylor, Groups with completely reducible regular representation, Proc. Amer. Math. Soc. 72 (1978), 593-600. MR 80b:22009 
[3] L. Baggett and K. F. Taylor, A sufficient condition for the complete reducibility of the regular representation, J. Funct. Anal. 34 (1979), 250-265. MR 81f:22005

[4] A. Belanger and B. Forrest, Geometric properties of coefficient function spaces determined by unitary representations of a locally compact group, J. Math. Anal. Appl. 193 (1995), 390-405. MR 96f:22005

[5] M. B. Bekka, A. T. Lau and G. Schlichting, On invariant subalgebras of the Fourier-Stieltjes algebra of a locally compact group, Math Ann. 294 (1992), 513-522. MR 93k:43006

[6] D. Bernier and K. F. Taylor, Wavelets from square-integrable representation, SIAM J. Math. Anal. 27 (1996), 594-608. MR 97h:22004

[7] L. C. Bunce, The Dunford-Pettis property in the predual of a von Neumann algebra, Proc. Amer. Math. Soc. 116 (1992), 99-100. MR 92k:46100

[8] R. B. Burckel, Weakly almost periodic functions on semigroups, Gordon and Breach, 1970. MR 41:8562

[9] C. H. Chu, A note on scattered $C^{*}$-algebras and the Radon-Nikodym property, J. London Math. Soc. 24 (1981), 533-536. MR 82k:46086

[10] L. Corwin and F. P. Greenleaf, Representations of nilpotent Lie groups and their applications. Part 1: Basic theory and examples, Cambridge, 1990. MR 92b:22007

[11] J. Dixmier, $C^{*}$-algebras, North-Holland, 1977. MR 56:16388

[12] J. Dixmier, Von Neumann algebras, North-Holland, 1981. MR 83a:46004

[13] R. Doss, On the transform of a singular or an absolutely continuous measure, Proc. Amer. Math. Soc. 19 (1968), 361-363. MR 36:5619

[14] M. Duflo and C. C. Moore, On the regular representation of a nonunimodular locally compact group, J. Funct. Anal. 21 (1976), 209-243. MR 52:14145

[15] P. Eymard, L'algèbre de Fourier d'un groupe localement compact, Bull. Soc. Math. France 92 (1964), 181-236. MR 37:4208

[16] J. M. G. Fell, Weak containment and induced representations of groups. II, Trans. Amer. Math. Soc. 110 (1964), 424-447. MR 28:3114

[17] V. Flory, On the Fourier algebra of a locally compact amenable group, Proc. Amer. Math. Soc. 29 (1971), 603-606. MR 44:371

[18] V. Flory, Eine Lebesgue-Zerlegung und funktorielle Eigenschaften der Fourier-Stieltjes Algebra, Inaugural Dissertation, Universität Heidelberg, 1972.

[19] E. E. Granirer and M. Leinert, On some topologies which coincide on the unit sphere of the Fourier-Stieltjes algebra $B(G)$ and the measure algebra $M(G)$, Rocky Mountain J. Math. 11 (1981), 459-472. MR 85f:43009

[20] F. P. Greenleaf, Amenable actions of locally compact groups, J. Funct. Anal. 4 (1969), 295315. MR 40:268

[21] S. Grosser and M. Moskowitz, Compactness conditions in topological groups, J. reine angew. Math. 246 (1971), 1-40. MR 44:1766

[22] C. Herz, Harmonic synthesis for subgroups, Ann. Inst. Fourier 23 (1973), 91-123. MR 50:7956

[23] E. Hewitt and K. A. Ross, Abstract harmonic analysis. II, Springer-Verlag, 1970. MR 41:7378

[24] R. Howe, The Fourier transform for nilpotent locally compact groups, Pacific J. Math. 73 (1977), 307-327. MR 58:11215

[25] E. Kaniuth, On primary ideals in group algebras, Monatsh. Math. 93 (1982), 293-302. MR 84i: 43003

[26] A. T. Lau, Closed convex invariant subsets of $L_{p}(G)$, Trans. Amer. Math. Soc. 232 (1977), 131-142. MR 57:17122

[27] A. T. Lau and V. Losert, The $C^{*}$-algebra generated by operators with compact support on a locally compact group, J. Funct. Anal. 112 (1993), 1-30. MR 94d:22005

[28] A. T. Lau and A. Ülger, Some geometric properties on the Fourier and Fourier-Stieltjes algebras of locally compact groups, Arens regularity and related problems, Trans. Amer. Math. Soc. 337 (1993), 321-359. MR 93g:22007

[29] P. F. Mah and T. Miao, Extreme points of the unit ball of the Fourier-Stieltjes algebra, Proc. Amer. Math. Soc. 128 (2000), 1097-1103. MR 2000i:43003

[30] T. Miao, Decomposition of $B(G)$, Trans. Amer. Math. Soc. 351 (1999), 4675-4692. MR 2000a: 43006

[31] C. C. Moore, Groups with finite dimensional irreducible representations, Trans. Amer. Math. Soc. 166 (1972), 401-410. MR 46:1960 
[32] R. D. Mosak, The $L^{1}$ - and $C^{*}$-algebras of $[F I A]_{B}^{-}$groups, and their representations, Trans. Amer. Math. Soc. 163 (1972), 277-310. MR 45:2096

[33] A. L. T. Paterson, Amenability, Mathematical Surveys and Monographs, Amer. Math. Soc., Providence, RI, 1988. MR 90e:43001

[34] J. P. Pier, Amenable locally compact groups, John Wiley and Sons, 1984. MR 86a:43001

[35] M. A. Rieffel, Unitary representations of group extensions. An algebraic approach to the theory of Mackey and Blattner, Adv. Math. Suppl. Stud. 4 (1979), 43-82. MR 81h:22004

[36] M. Takesaki, Theory of operator algebras.I, Springer-Verlag, 1979. MR 81e:46038

[37] K. F. Taylor, Geometry of Fourier algebras and locally compact groups with atomic decomposition, Math. Ann. 262 (1983), 183-190. MR 84h:43020

[38] C. R. Warner, Harmonic analysis on semi-simple Lie groups. II, Springer-Verlag, 1972. MR 58:16980

Fachbereich Mathematik/Informatik, Universität Paderborn, D-33095 Paderborn, Germany

E-mail address: kaniuth@math.uni-paderborn.de

Department of Mathematical and Statistical Sciences, University of Alberta, EdMONTON, CANADA T6G 2G1

E-mail address: tlau@math.ualberta.ca

Zentrum Mathematik, Technische Universität München, D-80290 München, Germany

E-mail address: schlicht@mathematik.tu-muenchen.de 